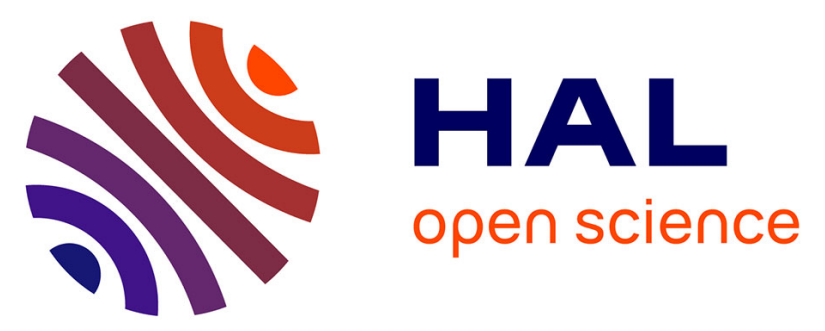

\title{
The Purcell Three-link swimmer: some geometric and numerical aspects related to periodic optimal controls
}

Piernicola Bettiol, Bernard Bonnard, Laetitia Giraldi, Pierre Martinon, Jérémy Rouot

\section{- To cite this version:}

Piernicola Bettiol, Bernard Bonnard, Laetitia Giraldi, Pierre Martinon, Jérémy Rouot. The Purcell Three-link swimmer: some geometric and numerical aspects related to periodic optimal controls. Variational Methods in Imaging and Geometric Control, 18, De Gruyter, pp.314-343, 2017, Radon Series on Computational and Applied Mathematics, 9783110439236. 10.1515/9783110430394-010 . hal-01143763v3

\section{HAL Id: hal-01143763 \\ https://hal.inria.fr/hal-01143763v3}

Submitted on 11 Mar 2016

HAL is a multi-disciplinary open access archive for the deposit and dissemination of scientific research documents, whether they are published or not. The documents may come from teaching and research institutions in France or abroad, or from public or private research centers.
L'archive ouverte pluridisciplinaire HAL, est destinée au dépôt et à la diffusion de documents scientifiques de niveau recherche, publiés ou non, émanant des établissements d'enseignement et de recherche français ou étrangers, des laboratoires publics ou privés. 


\title{
The Purcell Three-link swimmer: some geometric and numerical aspects related to periodic optimal controls
}

\author{
Piernicola Bettiol, Bernard Bonnard, Laetitia Giraldi, Pierre Martinon and \\ Jérémy Rouot
}

\begin{abstract}
The maximum principle combined with numerical methods is a powerful tool to compute solutions for optimal control problems. This approach turns out to be extremely useful in applications, including solving problems which require establishing periodic trajectories for Hamiltonian systems, optimizing the production of photobioreactors over a one-day period, finding the best periodic controls for locomotion models (e.g. walking, flying and swimming). In this article we investigate some geometric and numerical aspects related to optimal control problems for the so-called Purcell Three-link swimmer [20], in which the cost to minimize represents the energy consumed by the swimmer. More precisely, employing the maximum principle and shooting methods we derive optimal trajectories and controls, which have particular periodic features. Moreover, invoking a linearization procedure of the control system along a reference extremal, we estimate the conjugate points, which play a crucial role for the second order optimality conditions. We also show how, making use of techniques imported by the sub-Riemannian geometry, the nilpotent approximation of the system provides a model which is integrable, obtaining explicit expressions in terms of elliptic functions. This approximation allows to compute optimal periodic controls for small deformations of the body, allowing the swimmer to move minimizing its energy. Numerical simulations are presented using Hampath and Bocop codes.
\end{abstract}

Keywords. Periodic optimal controls, Purcell swimmer, First and second order necessary optimality conditions.

AMS classification. 49K15, 93C10, 70Q05.

\section{Introduction}

The study of periodic trajectories for Hamiltonian system represents a longstanding problem in dynamical systems and has attracted the interest of many researches, in particular for the $N$-body problem. The well-known Lyapunov-Poincaré theorem (cf. [6]) establishes, under suitable assumptions, the existence of a one-parameter family of periodic trajectories emanating from a given equilibrium point. The proof is

Work supported in part by the French Space Agency CNES, R\&T action R-S13/BS-005-012 and by the region Provence-Alpes-Côte d'Azur. Laetitia Giraldi was funded by the labex LMH through the grant ANR-11-LABX-0056-LMH in the "Programme des Investissements d'Avenir". 
based on the continuation method and leads to obtain periodic trajectories with small amplitudes. A different method to compute periodic trajectories was introduced by Poincaré investigating the $N$-body problem: this is the so-called direct method. The latter technique consists in finding a particular periodic trajectory which minimizes the action $S(x(t))=\int_{t_{0}}^{t_{1}} L(t, x(t), \dot{x}(t)) \mathrm{d} t$ (here $L$ is a given Lagrangian and $\left[t_{0}, t_{1}\right]$ is the time interval of reference), and which is a limit of a minimizing sequence (cf. [6]). The problem can be recast in the framework of optimal control theory, interpreting the derivative of $t \rightarrow x(t)$ as a control function $u($.$) . These two methods justify the use$ of a variational framework to compute periodic trajectories in optimal control, and, more precisely, a family of periodic trajectories depending on parameters such as the periods. The first-order necessary conditions for optimality, expressed in terms of the Euler-Lagrange equation in the context of the Calculus of Variations, are provided in optimal control by the maximum principle, which, due to the periodic structure of the problem, might detect a (parameterized) family of extremals. In these circumstances second-order analysis turns out to be an important tool in detecting the minimizers for the reference problem. Since the non-uniqueness of periodic minimizers do not allow in general to invoke standard second-order sufficient conditions, the necessity of refined second-order conditions was discussed in a series of articles (see for instance [22], [24],[10]), yielding important results which were tested in some 'academic' examples.

In control engineering, the importance of the study of periodic optimal controls is illustrated by the following problem areas: the optimization of the production of photobioreactors over a one-day prescribed time period (see for instance [14]), and, more recently, the search of periodic optimal controls in locomotion problems (e.g. walking, flying, swimming), where the state variable $x$ decomposes into two variables $\left(x^{\prime}, x^{\prime \prime}\right)$ where $x^{\prime}$ corresponds to the displacement variable and $x^{\prime \prime}$ stands for the shape variable (the latter must often satisfy periodic requirements in locomotion modeling). In the swimming problem, a swimmer displacement is produced by the deformation of the body interacting with the fluid and a periodic 'strategy of deformation' is called a stroke. In the case of micro-organisms evolving in a fluid, inertia is negligible with respect to the viscous effects, and the locomotion at this scale can be presented as a sub-Riemannian (SR) problem in which the cost functional to minimize represents the power expanded by the swimmer. A simplified mathematical model of swimmer is the Three linked spheres introduced by [17]. It turns out that the SR-geometry associated with this simplified model corresponds to the Heisenberg group case. This problem is equivalent to the Dido problem and the optimal solutions can be easily computed (cf. [5]). In the latter case, the optimal stroke are ellipses and they allow the swimmer to move along a desired direction [1]. An earlier pioneering model of micro-swimmer was introduced in the fifties in [23]; this was subsequently investigated using analytical tools coming from control theory in a recent paper [2].

In this article, we focus on the so-called Purcell Three-link swimmer [20]. By using the resistive force theory (see [13]), it was shown that the dynamics of the swimmer can 
be expressed explicitly in terms of an ordinary differential equation in which the speed of deformation can be interpreted as a control function (see for instance $[11,12]$ ). As a result, one obtains a drift-less control system which is linear with respect to the control variables, such as $\dot{x}=u_{1} F_{1}(x)+u_{2} F_{2}(x)$. Since the detailed expression of functions $F_{i}$ 's is quite involved, deriving the minimizers in an explicit form is not an easy task. In the present article we employ the expressions of the vector fields $F_{i}$ 's provided by previous work (cf. [2, 11, 12]) and, applying both geometric and numerical methods, we investigate the minimizers of our reference optimal control problem (modelling the Purcell swimmer) having some periodicity requirements.

The paper is organized as follows. Sections 2 is a short introduction to some tools and concepts imported from optimal control theory. These are subsequently employed for the study of some optimal control problems related to the mathematical model of the Purcell Three-link swimmer, which is described in Section 3. In Section 4, applying a classical SR-geometry approach, we provide an approximation associated with strokes of small amplitudes. This is the so-called nilpotent approximation and we show that it corresponds to the Cartan flat case [8],[21]. It turns out that the associated extremal curves are integrable in the class of elliptic functions. We provide detailed expressions of the extremals to make easier for the reader how to relate the period of the strokes to Jacobi complete integrals. Subsequently, Section 5 is devoted to the numerical analysis of the reference problem. More precisely, we estimate conjugate points for both normal and abnormal extremals, in relation with second order conditions. (In this context an open interesting question concerns the concept of focal point in relation with periodic optimal trajectories). Conjugate points are computed numerically using Hampath code. They are completed by numerical computations using Bocop code to evaluate strokes with general amplitudes using the system without any approximation and its energy function.

\section{First and second order optimality conditions}

First order necessary conditions for optimality (e.g. the Pontryagin maximum principle) and second order conditions play a crucial role in the selection and the characterization of solutions (minimizers) for problems in optimal control. Very general versions of first and second order optimality conditions are now available. Here, we restrict attention to optimal control problems with end-point constraints of the form

$$
\left\{\begin{array}{l}
\text { Minimize } \int_{0}^{T} L(x(t), u(t)) d t \\
\text { subject to } \\
\dot{x}(t)=f(x(t), u(t)) \quad \text { a.e. } t \in[0, T] \\
u(t) \in U \text { a.e. } t \in[0, T] \\
c(x(0), x(T))=0
\end{array}\right.
$$


in which $f(.,):. \mathbb{R}^{n} \times \mathbb{R}^{m} \rightarrow \mathbb{R}^{n}$ and $L(.,):. \mathbb{R}^{n} \times \mathbb{R}^{m} \rightarrow \mathbb{R}$ are given functions of class $\mathcal{C}^{2}$ with continuous second derivatives w.r.t. $(x, u)$ variables, $c(.,):. \mathbb{R}^{n} \times \mathbb{R}^{n} \rightarrow$ $\mathbb{R}^{\ell}$ is a given function of class $\mathcal{C}^{2}$ with continuous second derivatives w.r.t. $\left(x_{0}, x_{T}\right)$ variables, and $U \subset \mathbb{R}^{m}$ is a given set.

Take an optimal trajectory/control couple $(\bar{x}(),. \bar{u}()$.$) for (2.1). The Pontryagin$ maximum principle (see e.g. [19]) asserts (under appropriate hypotheses) that there exist a vector-valued function $p(.) \in W^{1,1}\left([0, T] ; \mathbb{R}^{n}\right)$, a vector $\nu \in \mathbb{R}^{\ell}$ and a constant $\lambda \geq 0$ such that

(i) $(p(),. \lambda) \neq(0,0)$ (The Nontriviality Condition),

(ii) $-\dot{p}(t)=p^{T}(t) \frac{\partial f}{\partial x}(\bar{x}(t), \bar{u}(t))-\lambda \frac{\partial L}{\partial x}(\bar{x}(t), \bar{u}(t)) \quad$ a.e.

(The Adjoint System),

(iii) $\langle p(t), f(\bar{x}(t), \bar{u}(t))\rangle-\lambda L(\bar{x}(t), \bar{u}(t))=\max _{u \in U}\{\langle p(t), f(\bar{x}(t), u)\rangle-$ $\lambda L(\bar{x}(t), u)\} \quad$ a.e.

(The Weierstrass or 'Maximization of the Hamiltonian' Condition), (iv) $\left[-p^{T}(0), p^{T}(T)\right]=\nu^{T} D_{x_{0}, x_{T}} c(\bar{x}(0), \bar{x}(T))$. (The Transversality Condition). Take a trajectory/control couple $(x(),. u()$.$) satisfying the control system of (2.1). If all$ the conditions (i)-(iv) of the Pontryagin maximum principle are satisfied for some absolutely continuous function $p($.$) , vector \nu \in \mathbb{R}^{\ell}$, and $\lambda \geq 0$, Then we call $(x(),. p()$. an extremal.

We shall consider the necessary conditions above both in the 'normal' and 'abnormal' form. 'Normal' means that the maximum principle is valid with the Lagrange multiplier $\lambda$ (associated with the objective function) different from zero (in this case it is not restrictive to take $\lambda=1 / 2$, by standard normalization). Whereas 'abnormal' means that the maximum principle applies with $\lambda=0$.

The pseudo Hamiltonian (also referred to as 'unmaximized' Hamiltonian) $\mathcal{H}: \mathbb{R}^{n} \times$ $\mathbb{R}^{n} \times \mathbb{R}^{m} \rightarrow \mathbb{R}^{m}$ is the function

$$
\mathcal{H}(x, p, u):=\langle p, f(x, u)\rangle-\lambda L(x, u) .
$$

If $\bar{u}(t)$ belongs to the interior of $U$ (this holds true whenever we take $U=\mathbb{R}^{m}$ ), condition (iii) above can be re-written in the form:

(iii) $^{\prime} \frac{\partial \mathcal{H}}{\partial u}(\bar{x}(t), p(t), \bar{u}(t))=0$.

Consider the particular case of (2.1) in which we impose partial periodic end-point constraints:

$$
x^{\prime}(0)=x_{0}^{\prime}, x^{\prime}(T)=x_{T}^{\prime}, x^{\prime \prime}(0)=x^{\prime \prime}(T),
$$

in which $x=\left(x^{\prime}, x^{\prime \prime}\right) \in \mathbb{R}^{k} \times \mathbb{R}^{n-k}$ for some fixed integer $0 \leq k<n$, and $x_{0}^{\prime}, x_{T}^{\prime} \in \mathbb{R}^{k}$ are given points. Then, $x^{\prime \prime}($.$) represents the periodic component of the$ state trajectory $x($.$) . Notice that the transversality condition (iv) involves only the com-$ ponent $p^{\prime \prime}($.$) of the adjoint arc p($.$) (which is associated with the 'periodic component'$ of a state arc $\bar{x}($.$\left.) , that is \bar{x}^{\prime \prime}().\right)$,

$$
p^{\prime \prime}(0)=p^{\prime \prime}(T) .
$$


Second order sufficient conditions for (local) optimality have been extensively investigated to derive optimal solutions with the property to be (locally) unique. This cannot be the case of pure periodic (i.e. when $k=0$ in problem (2.1) above) optimal control problems, in which given any periodic trajectory/control pair, any time translation produces a new periodic trajectory/control pair with the same cost. Therefore there is a growing interest in studying second order conditions in a framework which comprises periodic optimal control problems, and testing them in examples coming from applications (cf. [22], [24] and [10]).

The optimal control problem (2.1) above can be regarded as a sub-Riemannian problem whenever $f$ and $L$ have a particular structure:

$$
f(x, u)=\sum_{i=1}^{m} u_{i} F_{i}(x) \quad L(x, u)=\sum_{i=1}^{m} u_{i}^{2},
$$

where $\left\{F_{i}: \mathbb{R}^{n} \rightarrow \mathbb{R}^{n} \mid i=1, \ldots, m\right\}$ is a family smooth vector fields which is bracket generating. (In the representation above, for simplicity, we are also assuming that the vector fields $F_{i}$ 's are orthonormal.) In this case the integral cost $\int_{0}^{T} \sum_{i=1}^{m} u_{i}^{2}(t) d t$ represents the energy of a reference trajectory/control couple $(x(),. u()$. at a (given) final time $T$.

The concept of conjugate time (and conjugate point) plays a crucial role in optimality conditions, and can be characterized in terms of the degeneracy of the exponential mapping or, equivalently, of the quadratic form associated with the second variation of the endpoint mapping. A further important feature in the analysis of minimizers is represented by the notion of cut locus. We say that a point $\hat{x}$ is in the cut locus of a reference (left-end) point $x_{0}$ if we can find two minimizers joining $x_{0}$ and $\hat{x}$. It is well known that in Riemannian geometry every extremal is normal and $x($.$) is not a$ minimizer if and only if there exist a cut or a conjugate point along $x($.$) referred to the$ left end-point $x(0)$ (see for more details [6]).

\section{The Purcell Three-link swimmer}

\subsection{Mathematical Model}

Purcell's 3-link swimmer. The 3-link swimmer is modeled by the position of the center of the second stick $\mathbf{x}=(x, y)$, the angle $\theta$ between the $x$-axis and the second stick (the orientation of the swimmer). The shape of the swimmer defined by the two relative angles $\alpha_{1}$ and $\alpha_{2}$ (see Fig 1). We also denote by $L$ and $L_{2}$ the length of the two external arms and central link. In whats follows, $x^{\prime}$ (resp. $\left.x^{\prime \prime}\right)$ corresponds to $(x, y)$ and (resp. to $\left.\left(\theta, \alpha_{1}, \alpha_{2}\right)\right)$.

Dynamics via Resistive Force Theory. We approximate the non local hydrodynamic forces exerted by the fluid on the swimmer with local drag forces depending linearly on the velocity. We denote by $\mathbf{e}_{i}^{\|}$and $\mathbf{e}_{i}^{\perp}$ the unit vectors parallel and perpendicular to 


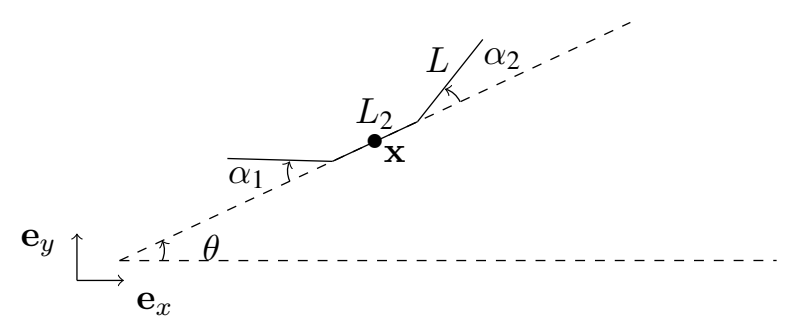

Figure 1: Purcell's 3-link swimmer.

the $i$-th link, and we also introduce $\mathbf{v}_{i}(s)$ the velocity of the point at distance $s$ from the extremity of the $i$-th link, that is

$$
\begin{aligned}
& \mathbf{v}_{1}(s)=\dot{\mathbf{x}}-\frac{L_{2}}{2} \dot{\theta} \mathbf{e}_{2}^{\perp}-s\left(\dot{\theta}-\dot{\alpha}_{1}\right) \mathbf{e}_{1}^{\perp}, \quad s \in[0, L], \\
& \mathbf{v}_{2}(s)=\dot{\mathbf{x}}-\left(s-\frac{L_{2}}{2}\right) \dot{\theta} \mathbf{e}_{2}^{\perp}, \quad s \in\left[0, L_{2}\right], \\
& \mathbf{v}_{3}(s)=\dot{\mathbf{x}}+\frac{L_{2}}{2} \dot{\theta} \mathbf{e}_{2}^{\perp}+s\left(\dot{\theta}-\dot{\alpha}_{2}\right) \mathbf{e}_{3}^{\perp}, \quad s \in[0, L] .
\end{aligned}
$$

The force $\mathbf{f}_{i}$ acting on the $i$-th segment is taken as

$$
\mathbf{f}_{i}(s):=-\xi\left(\mathbf{v}_{i}(s) \cdot \mathbf{e}_{i}^{\|}\right) \mathbf{e}_{i}^{\|}-\eta\left(\mathbf{v}_{i}(s) \cdot \mathbf{e}_{i}^{\perp}\right) \mathbf{e}_{i}^{\perp},
$$

where $\xi$ and $\eta$ are respectively the drag coefficients in the directions of $\mathbf{e}_{i}^{\|}$and $\mathbf{e}_{i}^{\perp}$. Neglecting inertia forces, Newton laws are written as

$$
\left\{\begin{array}{l}
\mathbf{F}=0 \\
\mathbf{e}_{z} \cdot \mathbf{T}_{\mathbf{x}}=0
\end{array}\right.
$$

where $\mathbf{F}$ is the total force exerted on the swimmer by the fluid and $\mathbf{e}_{z}=\mathbf{e}_{x} \wedge \mathbf{e}_{y}$,

$$
\mathbf{F}=\int_{0}^{L} \mathbf{f}_{1}(s) d s+\int_{0}^{L_{2}} \mathbf{f}_{2}(s) d s+\int_{0}^{L} \mathbf{f}_{3}(s) d s
$$

and $\mathbf{T}_{\mathbf{x}}$ is the corresponding total torque computed with respect to the central point $\mathbf{x}$,

$$
\begin{array}{r}
\mathbf{T}_{\mathbf{x}}=\int_{0}^{L}\left(\mathbf{x}_{1}(s)-\mathbf{x}_{1}\right) \times \mathbf{f}_{1}(s) d s+\int_{0}^{L_{2}}\left(\mathbf{x}_{2}(s)-\mathbf{x}_{1}\right) \times \mathbf{f}_{2}(s) d s \\
+\int_{0}^{L}\left(\mathbf{x}_{3}(s)-\mathbf{x}_{1}\right) \times \mathbf{f}_{3}(s) d s .
\end{array}
$$


Since the $\mathbf{f}_{i}(s)$ are linear in $\dot{\mathbf{x}}, \dot{\theta}, \dot{\alpha}_{1}, \dot{\alpha}_{2}$, the system (3.1) can be rewritten as

$$
\mathbf{A}(z) \cdot\left(\begin{array}{c}
\dot{\mathbf{x}} \\
\dot{\theta}
\end{array}\right)-\mathbf{B}(z) \cdot\left(\begin{array}{c}
\dot{\alpha}_{1} \\
\dot{\alpha}_{2}
\end{array}\right)=0
$$

where $z(t):=\left(\alpha_{1}, \alpha_{2}, x, y, \theta\right)(t)^{T}$. The matrix $\mathbf{A}(z)$ is known as the "Grand Resistance Matrix" and is invertible (see [2]). Then the dynamics of the swimmer is finally expressed as an ODE system

$$
\dot{z}(t)=f\left(z, \dot{\alpha}_{1}, \dot{\alpha}_{2}\right)=\dot{\alpha}_{1}(t) F_{1}(z(t))+\dot{\alpha}_{2}(t) F_{2}(z(t)),
$$

where $\left(\begin{array}{ll}F_{1}(z) & F_{2}(z)\end{array}\right):=\left(\begin{array}{c}\mathbb{I}_{2} \\ \mathbf{A}^{-1}(z) \mathbf{B}(z)\end{array}\right)$ with $\mathbb{I}_{2}$ the $2 \times 2$ identity matrix. The detailed expression for the $F_{i}$ is quite complicated and takes several pages (see e.g. $[2,11,12])$.

At the end, the dynamics of the swimmer is governed by an ordinary differential equation linear with respect to the speed of deformation, $\dot{\alpha}_{i}, i=1,2$. By considering the latter as a control function, $u_{i}:=\dot{\alpha}_{i}, i=1,2$, we then obtain an linear control problem without drift.

By definition, the power expanded during a time $T>0$ by the swimmer is given by (see [1] for more details)

$$
\int_{0}^{T}\left(\int_{0}^{L} \mathbf{f}_{1} \cdot \mathbf{v}_{1}+\int_{0}^{L_{2}} \mathbf{f}_{2} \cdot \mathbf{v}_{2}+\int_{0}^{L} \mathbf{f}_{3} \cdot \mathbf{v}_{3}\right) .
$$

Notice that the power is then a quadratic function with respect to the speed of deformation of the body.

\section{Local analysis for the three-link Purcell swimmer}

The sub-Riemannnian structure of the Purcell swimmer model allows to consider a motion of first-order approximation which takes into account the non-isotropic behaviour of the sub-Riemannian distance, called nilpotent approximation. This approximation is called nilpotent in the sense that the vector fields $F_{1}$ and $F_{2}$ can be approximated (using new coordinates, called privileged coordinates) by vector fields $\hat{F}_{1}$ and $\hat{F}_{2}$ which generate a nilpotent Lie algebra. The nilpotent approximation with the accompanying privileged coordinates constitutes the basis for the infinitesimal calculus adapted to the particular structure of the (non honolomic) control system modelling the Purcell swimmer. We refer the reader for these constructs for instance to [3].

\subsection{Computations of the nilpotent approximation}

Let us denote $D=\operatorname{span}\left\{\hat{F}_{1}, \hat{F}_{2}\right\}, D_{1}=D, D_{2}=\operatorname{span}\left\{D_{1} \cup\left[D_{1}, D_{2}\right]\right\}$ and $D_{3}=$ $\operatorname{span}\left\{D_{2} \cup\left[D_{1}, D_{2}\right]\right\}$. At the point $x_{0}$ we have a $(2,3,5)$-distribution corresponding 
to the respective rank of $D_{1}, D_{2}$ and $D_{3}$.

We write the control system as $\dot{x}=F u=\sum_{i=1}^{2} u_{i} F_{i}$.

\section{Feedback group}

The pseudo-group $\mathcal{G}=(\varphi, \beta)$ is defined by the actions :

- local diffeomorphism $\varphi$ :

let $\dot{x}=X(x)$ and $x=\varphi(y)$.

The action of $\varphi$ on a vector field $X$ is $\dot{y}=(\varphi * X)(y)=\left[\frac{\partial \varphi}{\partial y}^{-1} X o \varphi\right](y)$, $\varphi * F=\left(\varphi * F_{1}, \varphi * F_{2}\right)$.

- feedback $\beta$ :

$u=\beta(x) v$ where $\beta$ is a $2 \times 2$ invertible matrix. The action of $\beta$ transforms $F$ into $F \beta$.

\section{Computations}

Let us define the variables $x_{i} i=1, \cdots, 5$ as $x_{1}=\alpha_{1}, x_{2}=\alpha_{2},\left(x_{3}, x_{4}\right)=\mathbf{x}, x_{5}=\theta$. In the rest of the computations, we set $L=1, L_{2}=2, \xi=1, \eta=2$.

Hence, the 2-jets of $F_{1}$ and $F_{2}$ at zero are expressed by

$$
\begin{aligned}
F_{1}(x) & =\frac{\partial}{\partial x_{1}}+\left(-\frac{1}{6} x_{5}-\frac{4}{27} x_{1}-\frac{2}{27} x_{2}\right) \frac{\partial}{\partial x_{3}} \\
& +\left(\frac{1}{6}-\frac{1}{12} x_{5}^{2}-\frac{2}{27} x_{5} x_{2}-\frac{4}{27} x_{5} x_{1}-\frac{1}{27} x_{1}^{2}-\frac{1}{27} x_{1} x_{2}-\frac{1}{36} x_{2}^{2}\right) \frac{\partial}{\partial x_{4}} \\
& +\left(-\frac{7}{27}+\frac{2}{81} x_{1}^{2}-\frac{2}{81} x_{1} x_{2}-\frac{5}{162} x_{2}^{2}\right) \frac{\partial}{\partial x_{5}}+O\left(|x|^{3}\right) \\
F_{2}(x) & =\frac{\partial}{\partial x_{2}}+\left(\frac{1}{6} x_{5}+\frac{4}{27} x_{2}+\frac{2}{27} x_{1}\right) \frac{\partial}{\partial x_{3}} \\
& +\left(-\frac{1}{6}+\frac{1}{12} x_{5}^{2}+\frac{4}{27} x_{5} x_{2}+\frac{2}{27} x_{5} x_{1}+\frac{1}{36} x_{1}^{2}+\frac{1}{27} x_{1} x_{2}+\frac{1}{27} x_{2}^{2}\right) \frac{\partial}{\partial x_{4}} \\
& +\left(-\frac{7}{27}-\frac{5}{162} x_{1}^{2}-\frac{2}{81} x_{1} x_{2}+\frac{2}{81} x_{2}^{2}\right) \frac{\partial}{\partial x_{5}}+O\left(|x|^{3}\right)
\end{aligned}
$$

The normal forms fo these mappings (see [21]) are

$$
\left(\varphi * F_{1}\right)=\frac{\partial}{\partial x_{1}}+O\left(|x|^{3}\right), \quad\left(\varphi * F_{2}\right)=\frac{\partial}{\partial x_{2}}+x_{1} \frac{\partial}{\partial x_{3}}+\frac{\partial}{\partial x_{4}}+x_{1}^{2} \frac{\partial}{\partial x_{5}}+O\left(|x|^{3}\right) .
$$

We introduce the weights 1 for $x_{1}, x_{2}, 2$ for $x_{3}$ and 3 for $x_{4}, x_{5}$. If $x_{i}$ is of order $p, \frac{\partial}{\partial x_{i}}$ is of order $-p$ to define the nilpotent normal form of order -1 .

We write $\varphi=\varphi_{N} \circ \ldots o \varphi_{1}: \mathbb{R}^{5} \rightarrow \mathbb{R}^{5}$. At each step $i$, for $i=1, \ldots, N$ of the computations we shall use $N=13$ steps : 
. $x=\left(x_{1}, x_{2}, x_{3}, x_{4}, x_{5}\right)$ are the old local coordinates and $y=\left(y_{1}, y_{2}, y_{3}, y_{4}, y_{5}\right)$ the new ones resulting from the change of variables $\varphi_{i}$,

. $x_{j}=\varphi_{i}^{(j)}\left(y_{j}\right): \mathbb{R} \rightarrow \mathbb{R}$ denoting the $\mathrm{j}^{\text {th }}$ component of $\varphi_{i}$ for some $j \in\{1, \ldots, 5\}$. The other components $\varphi_{i}^{(k)}, k \neq j$ are the identity transformations.

The successive change of variables are given by

1. $x_{5}=\varphi_{1}^{(5)}\left(y_{5}\right)=y_{5}-\frac{7}{27} y_{1}$,

2. $x_{3}=\varphi_{2}^{(3)}\left(y_{3}\right)=y_{3}-\frac{1}{6} y_{5} y_{1}-\frac{17}{324} y_{1}^{2}-\frac{2}{27} y_{2} y_{1}$,

3. $x_{4}=\varphi_{3}^{(4)}\left(y_{4}\right)=y_{4}+\frac{1}{6} y_{1}-\frac{37}{3 \times 8748} y_{1}^{3}$,

4. $x_{5}=\varphi_{4}^{(5)}\left(y_{5}\right)=y_{5}+\frac{2}{24} y_{1}^{3}-\frac{2}{162}$,

5. $x_{5}=\varphi_{5}^{(5)}\left(y_{5}\right)=y_{5}-\frac{7}{27} y_{2}$,

6. $x_{3}=\varphi_{6}^{(3)}\left(y_{3}\right)=\frac{5}{81} y_{3}+\frac{17}{324} y_{2}^{2}+\frac{1}{6} y_{5} y_{2}$,

7. $x_{4}=\varphi_{7}^{(4)}\left(y_{4}\right)=y_{4}-y_{3} y_{2}$,

8. $x_{4}=\varphi_{8}^{(4)}\left(y_{4}\right)=y_{4}+\frac{37}{3 \times 8748} y_{2}^{3}$,

9. $x_{4}=\varphi_{9}^{(4)}\left(y_{4}\right)=y_{4}-54 \times \frac{83}{8748} y_{5}$,

10. $x_{4}=\varphi_{10}^{(4)}\left(y_{4}\right)=y_{4}+\frac{2270}{2187} y_{2} y_{3}+\frac{5}{81} y_{5} y_{3}+\frac{83}{3 \times 6561} y_{2}^{3}$,

11. $x_{4}=\varphi_{11}^{(4)}\left(y_{4}\right)=-\frac{83}{2187} y_{4}$,

12. $x_{5}=\varphi_{12}^{(5)}\left(y_{5}\right)=y_{5}+\frac{1}{27} y_{3} y_{2}+\frac{2}{3 \times 81} y_{2}^{3}$,

13. $x_{5}=\varphi_{13}^{(5)}\left(y_{5}\right)=-\frac{1}{54} y_{5}-\frac{1}{27} y_{4}$.

Neglecting terms of order greater than 3 , we denote by $\hat{F}_{1}, \hat{F}_{2}$ the resulting vector fields.

Remark 4.1. The construction of the diffeomorphism relates the normalized coordinates to the physical coordinates. A similar transformation details the effect on a frame.

\subsection{Integration of extremal trajectories}

For two vector fields $F$ and $G$, we use the following Lie bracket convention

$$
[F, G](x)=\frac{\partial F}{\partial x}(x) G(x)-\frac{\partial G}{\partial x}(x) F(x)
$$

Computing we have

$$
\hat{F}_{1}(x)=\frac{\partial}{\partial x_{1}}, \quad \hat{F}_{2}(x)=\frac{\partial}{\partial x_{2}}+x_{1} \frac{\partial}{\partial x_{3}}+x_{3} \frac{\partial}{\partial x_{4}}+x_{1}^{2} \frac{\partial}{\partial x_{5}},
$$




$$
\begin{aligned}
& {\left[\hat{F}_{1}, \hat{F}_{2}\right](x)=-\frac{\partial}{\partial x_{3}}-2 x_{1} \frac{\partial}{\partial x_{5}}, \quad\left[\left[\hat{F}_{1}, \hat{F}_{2}\right], \hat{F}_{1}\right](x)=-2 \frac{\partial}{\partial x_{5}}} \\
& {\left[\left[\hat{F}_{1}, \hat{F}_{2}\right], \hat{F}_{2}\right](x)=\frac{\partial}{\partial x_{4}} .}
\end{aligned}
$$

All brackets of length greater than 3 are zero.

We introduce

$$
\begin{array}{ll}
H_{1}=\left\langle p, \hat{F}_{1}(x)\right\rangle=p_{1}, & H_{2}=\left\langle p, \hat{F}_{2}(x)\right\rangle=p_{2}+p_{3} x_{1}+p_{4} x_{3}+p_{5} x_{1}^{2}, \\
H_{3}=\left\langle p,\left[\hat{F}_{1}, \hat{F}_{2}\right](x)\right\rangle=-p_{3}-2 x_{1} p_{5}, & H_{4}=\left\langle p,\left[\left[\hat{F}_{1}, \hat{F}_{2}\right], \hat{F}_{1}\right](x)\right\rangle=-2 p_{5}, \\
H_{5}=\left\langle p,\left[\left[\hat{F}_{1}, \hat{F}_{2}\right], \hat{F}_{2}\right](x)\right\rangle=p_{4} . &
\end{array}
$$

We recall the following relation for the Poisson brackets of two lifting Hamiltonians $H_{F}$ and $H_{G}$ of vector fields $F$ and $G$.

If

$$
H_{F}=\langle p, F(x)\rangle, \quad H_{G}=\langle p, G(x)\rangle,
$$

are the Hamiltonian lifts of vector fields of $F$ and $G$, then we have

$$
\left\{H_{F}, H_{G}\right\}=\langle p,[F, G](x)\rangle \text {. }
$$

We consider the SR-Cartan flat case [21], [8]

$$
\dot{x}=\sum_{i=1}^{2} u_{i} F_{i}, \quad \min _{u} \int_{0}^{T}\left(u_{1}^{2}+u_{2}^{2}\right) d t .
$$

Normal case. The pseudo Hamiltonian is

$$
\mathcal{H}=\sum_{i} u_{i} H_{i}-\frac{1}{2}\left(u_{1}^{2}+u_{2}^{2}\right)
$$

The Pontryagin maximum principle [19] gives $u_{i}=H_{i}$.

Hence, the true Hamiltonian is

$$
H=\frac{1}{2}\left(H_{1}^{2}+H_{2}^{2}\right)
$$

Computing we have

$$
\begin{array}{ll}
\dot{H}_{1}=d H_{1}(\vec{H})=\left\{H_{1}, H_{2}\right\} H_{2}=\left\langle p,\left[\hat{F}_{1}, \hat{F}_{2}\right](x)\right\rangle H_{2}=H_{2} H_{3}, & \\
\dot{H}_{2}=-H_{3} H_{1}, & \dot{H}_{3}=H_{1} H_{4}+H_{2} H_{5}, \\
\dot{H}_{4}=0 \quad \text { hence } \quad H_{4}=c_{4}, \quad \dot{H}_{5}=0 \text { hence } H_{5}=c_{5} .
\end{array}
$$


Fixing the level energy, $H_{1}^{2}+H_{2}^{2}=1$ we set $H_{1}=\cos (\theta)$ and $H_{2}=\sin (\theta)$.

$$
\dot{H}_{1}=-\sin (\theta) \dot{\theta}=H_{2} H_{3}=\sin (\theta) H_{3} .
$$

Hence $\dot{\theta}=-H_{3}$ and

$$
\ddot{\theta}=-\left(H_{1} c_{4}+H_{2} c_{5}\right)=-c_{4} \cos (\theta)-c_{5} \sin (\theta)=-A \sin (\theta+\phi)
$$

where $A$ is a constant.

By identification, we get $A \sin (\phi)=c_{4}$ and $A \cos (\phi)=c_{5}$.

Let $\psi=\theta+\phi$, we get

$$
\frac{1}{2} \dot{\psi}^{2}-A \cos (\psi)=B
$$

where $B$ is a constant.

We have the two following cases :

Oscillating case

$$
\dot{\psi}^{2}=4 A\left(\frac{1}{2}+\frac{B}{2 A}-\sin ^{2}(\psi / 2)\right) .
$$

We introduce $\omega^{2}=A$ and $k^{2}=\frac{1}{2}+\frac{B}{2 A}$ with $0<k<1$, and we obtain [16]

$$
\sin (\psi / 2)=k \operatorname{sn}(u, k), \quad \cos (\psi / 2)=\operatorname{dn}(u, k)
$$

where $u=\omega t+\varphi_{0}$.

$H_{1}$ and $H_{2}$ are elliptic functions of the first kind. Therefore the system becomes

$$
\begin{array}{ll}
\dot{x}_{1}=H_{1}, & \dot{x}_{2}=H_{2}, \quad \dot{x}_{3}=H_{2} x_{1}, \\
\dot{x}_{4}=H_{2} x_{3}, \quad & \dot{x}_{5}=H_{2} x_{1}^{2} .
\end{array}
$$

Parameterizing (4.4) with respect to $u$ we have

$$
\begin{aligned}
\frac{\mathrm{d} x_{1}}{\mathrm{~d} u}= & \frac{1}{\omega} \cos \left(\theta\left(\left(u-\varphi_{0}\right) / \omega\right)\right)=\frac{1}{\omega}\left[2 k \sin (\phi) \operatorname{sn}(u) \mathrm{dn}(u)+\left(2 \mathrm{dn}^{2}(u)-1\right) \cos (\phi)\right] . \\
\frac{\mathrm{d} x_{2}}{\mathrm{~d} u} & =\frac{1}{\omega} \sin \left(\theta\left(\left(u-\varphi_{0}\right) / \omega\right)\right)=\frac{1}{\omega}\left[2 k \cos (\phi) \operatorname{sn}(u) \operatorname{dn}(u)+\left(1-2 \mathrm{dn}^{2}(u)\right) \sin (\phi)\right] . \\
\frac{\mathrm{d} x_{3}}{\mathrm{~d} u} & =\frac{1}{\omega} \sin \left(\theta\left(\left(u-\varphi_{0}\right) / \omega\right)\right) x_{1}\left(\left(u-\varphi_{0}\right) / \omega\right) \\
& =\frac{1}{\omega^{2}}\left[-4 k^{2} \sin (\phi) \cos (\phi) \operatorname{cn}(u) \operatorname{sn}(u) \mathrm{dn}(u)+2 E(u)\right) \sin (\phi) \cos (\phi) \\
& +2 x_{1}\left(\varphi_{0}\right) k \cos (\phi) \operatorname{sn}(u) \operatorname{dn}(u)+\left(-2 \mathrm{dn}^{2}(u)+1\right) x_{1}\left(\varphi_{0}\right) \sin (\phi) \\
& +\left(-2 u \operatorname{sn}(u) \mathrm{dn}(u)-4 \operatorname{cn}(u) \mathrm{dn}^{2}(u)+4 \operatorname{sn}(u) \mathrm{dn}(u) E(u)\right. \\
& +2 \operatorname{cn}(u)) k \cos ^{2}(\phi)+\left(2 u \mathrm{dn}^{2}(u)-4 \mathrm{dn}^{2}(u) E(u)-u\right. \\
& \left.+\left(4 \operatorname{cn}(u) \mathrm{dn}^{2}(u)-2 \operatorname{cn}^{2}(u)\right) k\right] .
\end{aligned}
$$




$$
\begin{aligned}
& \frac{\mathrm{d} x_{4}}{\mathrm{~d} u}=\frac{1}{\omega} \sin \left(\theta\left(\left(u-\varphi_{0}\right) / \omega\right)\right) x_{3}\left(\left(u-\varphi_{0}\right) / \omega\right) \\
& =\frac{1}{\omega^{3}}\left[4 k^{4} \cos ^{3}(\phi) \operatorname{sn}^{4}(u)-8 k^{4} \cos (\phi) \operatorname{sn}^{4}(u)\right. \\
& -4 k^{2} x_{1}\left(\varphi_{0}\right) \cos ^{2}(\phi) \operatorname{cn}(u) \operatorname{sn}(u) \operatorname{dn}(u)+2 x_{3}\left(\varphi_{0}\right) k \cos (\phi) \operatorname{sn}(u) \operatorname{dn}(u) \\
& +4 k^{3} \sin (\phi) \operatorname{sn}(u) \operatorname{dn}(u)+\left(-u^{2} \operatorname{sn}(u) \operatorname{dn}(u)-4 u \operatorname{cn}(u) \operatorname{dn}^{2}(u)\right. \\
& +4 u \operatorname{sn}(u) \operatorname{dn}(u) E(u)+8 \operatorname{cn}(u) \operatorname{dn}^{2}(u) E(u)-4 \operatorname{sn}(u) \operatorname{dn}(u) E(u)^{2} \\
& +2 u \operatorname{cn}(u)-4 \operatorname{cn}(u) E(u)-2 \operatorname{sn}(u) \operatorname{dn}(u)) k \sin (\phi) \\
& +(4 u \operatorname{cn}(u) \operatorname{sn}(u) \operatorname{dn}(u)-8 \operatorname{cn}(u) \operatorname{sn}(u) \operatorname{dn}(u) E(u) \\
& \left.+4 \mathrm{dn}^{2}(u)-2\right) k^{2} \cos ^{3}(\phi)+\left(-2 x_{3}\left(\varphi_{0}\right) \mathrm{dn}^{2}(u)+x_{3}\left(\varphi_{0}\right)\right) \sin (\phi) \\
& +\left(u^{2} \operatorname{sn}(u) \operatorname{dn}(u)+4 u \operatorname{cn}(u) \operatorname{dn}^{2}(u)-4 u \operatorname{sn}(u) \operatorname{dn}(u) E(u)\right. \\
& -8 \mathrm{cn}(u) \mathrm{dn}^{2}(u) E(u)+4 \operatorname{sn}(u) \operatorname{dn}(u) E(u)^{2}-2 u \operatorname{cn}(u) \\
& +4 \operatorname{cn}(u) E(u)) k \sin ^{3}(\phi)+\left(2 u \mathrm{dn}^{2}(u)-4 \mathrm{dn}^{2}(u) E(u)-u\right. \\
& +2 E(u)) x_{1}\left(\varphi_{0}\right) \cos ^{2}(\phi)+\left(-u^{2} \operatorname{dn}^{2}(u)+4 u \operatorname{dn}^{2}(u) E(u)\right. \\
& \left.-4 \mathrm{dn}^{2}(u) E(u)^{2}+1 / 2 u^{2}-2 u E(u)+2 \mathrm{dn}^{2}(u)+2 E(u)^{2}-2\right) \cos ^{3}(\phi) \\
& +\left(u^{2} \operatorname{dn}^{2}(u)-4 u \operatorname{dn}^{2}(u) E(u)+4 \operatorname{dn}^{2}(u) E(u)^{2}\right. \\
& \left.-1 / 2 u^{2}+2 u E(u)-6 \mathrm{dn}^{2}(u)-2 E(u)^{2}+6\right) \cos (\phi) \\
& +\left(2 u \operatorname{sn}(u) \operatorname{dn}(u)+4 \operatorname{cn}(u) \operatorname{dn}^{2}(u)-4 \operatorname{sn}(u) \operatorname{dn}(u) E(u)\right. \\
& -2 \mathrm{cn}(u)) \cos (\phi) k x_{1}\left(\varphi_{0}\right) \sin (\phi)+\left(-4 \operatorname{dn}^{2}(u)+2\right) k^{2} \cos (\phi) \\
& +\left(4 \operatorname{sn}^{3}(u) \operatorname{dn}(u)-4 \operatorname{sn}(u) \operatorname{dn}(u)\right) k^{3} \sin ^{3}(\phi) \\
& \left.+\left(-2 u \operatorname{dn}^{2}(u)+4 \operatorname{dn}^{2}(u) E(u)+u-2 E(u)\right) x_{1}\left(\varphi_{0}\right)\right] .
\end{aligned}
$$

$$
\begin{aligned}
\frac{\mathrm{d} x_{5}}{\mathrm{~d} u} & =\frac{1}{\omega} \sin \left(\theta\left(\left(u-\varphi_{0}\right) / \omega\right)\right) x_{1}\left(\left(u-\varphi_{0}\right) / \omega\right)^{2} \\
& =\frac{1}{\omega^{3}}\left[-8 k^{4} \sin ^{3}(\phi) \operatorname{sn}^{4}(u)-8 k^{2} x_{1}\left(\varphi_{0}\right) \sin (\phi) \cos (\phi) \operatorname{cn}(u) \operatorname{sn}(u) \mathrm{dn}(u)\right. \\
& +2 x_{1}\left(\varphi_{0}\right)^{2} k \cos (\phi) \operatorname{sn}(u) \operatorname{dn}(u)+(-8 u \operatorname{cn}(u) \operatorname{sn}(u) \operatorname{dn}(u) \\
& \left.+16 \operatorname{cn}(u) \operatorname{sn}(u) \operatorname{dn}(u) E(u)-8 \operatorname{dn}^{2}(u)+4\right) k^{2} \sin ^{3}(\phi) \\
& +\left(4 u \operatorname{dn}^{2}(u)-8 \operatorname{dn}^{2}(u) E(u)-2 u+4 E(u)\right) x_{1}\left(\varphi_{0}\right) \sin (\phi) \cos (\phi)
\end{aligned}
$$




$$
\begin{aligned}
& +\left(-2 u^{2} \operatorname{dn}^{2}(u)+8 u \operatorname{dn}^{2}(u) E(u)-8 \operatorname{dn}^{2}(u) E(u)^{2}\right. \\
& \left.+u^{2}-4 u E(u)+4 E(u)^{2}\right) \sin (\phi)+\left(-2 \operatorname{dn}^{2}(u)+1\right) x_{1}\left(\varphi_{0}\right)^{2} \sin (\phi) \\
& +(8 u \operatorname{cn}(u) \operatorname{sn}(u) \operatorname{dn}(u)-16 \operatorname{cn}(u) \operatorname{sn}(u) \operatorname{dn}(u) E(u)) k^{2} \sin (\phi) \\
& +\left(2 u^{2} \operatorname{sn}(u) \operatorname{dn}(u)+8 u \operatorname{cn}(u) \operatorname{dn}^{2}(u)-8 u \operatorname{sn}(u) \operatorname{dn}(u) E(u)\right. \\
& -16 \operatorname{cn}(u) \operatorname{dn}^{2}(u) E(u)+8 \operatorname{sn}(u) \operatorname{dn}(u) E(u)^{2}-4 u \operatorname{cn}(u) \\
& +8 \operatorname{cn}(u) E(u)) k \cos ^{3}(\phi)+\left(8 \operatorname{sn}^{3}(u) \operatorname{dn}(u)-8 \operatorname{sn}(u) \operatorname{dn}(u)\right) k^{3} \cos ^{3}(\phi) \\
& +\left(-4 u \operatorname{sn}(u) \operatorname{dn}(u)-8 \operatorname{cn}(u) \operatorname{dn}^{2}(u)+8 \operatorname{sn}(u) \operatorname{dn}(u) E(u)\right. \\
& +4 \operatorname{cn}(u)) x_{1}\left(\varphi_{0}\right) k \cos ^{2}(\phi)+\left(-8 u \operatorname{cn}(u) \operatorname{dn}^{2}(u)-8 \operatorname{cn}(u) E(u)\right. \\
& +4 u \operatorname{cn}(u)-8 \operatorname{cn}(u) E(u)) k \cos ^{2}(\phi)+\left(-8 \operatorname{sn}^{3}(u) \operatorname{dn}(u)\right. \\
& \left.+8 \operatorname{sn}(u) \operatorname{dn}^{2}(u)\right) k^{3} \cos (\phi)+\left(2 u^{2} \operatorname{dn}^{2}(u)-8 u \operatorname{dn}^{2}(u) E(u)\right. \\
& \left.+8 \operatorname{dn}^{2}(u) E(u)^{2}-u^{2}+4 u E(u)-4 \operatorname{dn}^{2}(u)-4 E(u)^{2}+4\right) \sin ^{3}(\phi) \\
& \left.+\left(8 \operatorname{cn}^{2}(u) \operatorname{dn}^{2}(u)-4 \operatorname{cn}^{2}(u)\right) x_{1}\left(\varphi_{0}\right) k\right] .
\end{aligned}
$$

Proposition 4.2. The solution $x(u)$ of the system (4.4) can be expressed as a polynomial function of $(u, \operatorname{sn}(u), \operatorname{cn}(u), \operatorname{dn}(u), E(u))$.

Proof. Integrating equations (4.5) to (4.2) thanks to formulae (4.9) gives the result.

\section{Remark 4.3. [16]}

- sn, cn are $4 K$-periodics,

- $\mathrm{dn}$ is $2 K$-periodic,

- $E(u)=\frac{E}{K} u+Z(u)$ where $E, K$ are complete integrals and $Z$ is the $2 K$-periodic zeta function.

The next step is to compute the $x$ variables using quadratures in the oscillating case. Since $x(0)=0$, solutions depend upon 4 independent parameters $H_{i}(0)$ for $i=1, \ldots, 5$ coupled with the relation $H_{1}(t=0)^{2}+H_{2}(t=0)^{2}=1$.

To integrate the equations (4.5)-(4.2) explicitly, we use the following primitive functions (see [16])

$$
\begin{aligned}
& \int \operatorname{dn}^{2}(u) d u=E(u), \quad \int \operatorname{cn}(u) \operatorname{dn}(u) d u=\operatorname{sn}(u), \quad \int \operatorname{cn}(u) \operatorname{sn}(u) \operatorname{dn}(u) d u=-\frac{1}{2} \operatorname{cn}(u)^{2}, \\
& \int \operatorname{cn}(u) d u=\frac{1}{k} \arctan \left(k \frac{\operatorname{sn}(u)}{\operatorname{dn}(u)}\right), \quad \int u \operatorname{sn}(u) \operatorname{dn}(u) d u=\frac{1}{k} \arctan \left(k \frac{\operatorname{sn}(u)}{\operatorname{dn}(u)}\right)-u \operatorname{cn}(u), \\
& \int E(u) \operatorname{sn}(u) \operatorname{dn}(u) d u=-E(u) \operatorname{cn}(u)+\frac{1}{2 k} \arctan \left(k \frac{\operatorname{sn}(u)}{\operatorname{dn}(u)}\right)+\frac{1}{2} \operatorname{dn}(u) \operatorname{sn}(u),
\end{aligned}
$$




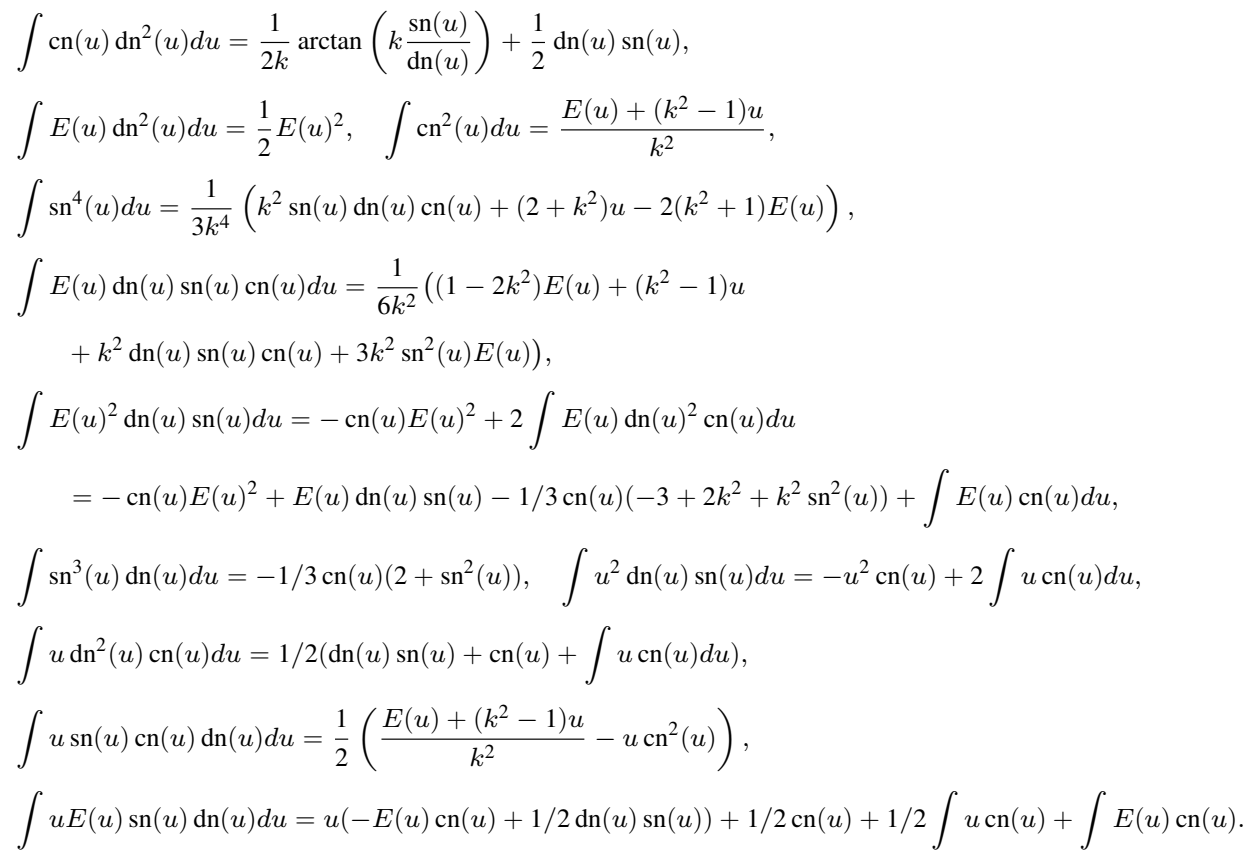

The final expressions of the solution $\left(x_{i}(u)\right)_{i=1, \ldots, 5}$ of (4.4). (we supply a MAPLE code to check the correctness of the expressions)

- $x_{1}(u)=\frac{1}{\omega}\left[x_{1}\left(\varphi_{0}\right)-2 k \sin (\phi) \operatorname{cn}(u)+(-u+2 E(u)) \cos (\phi)\right]$.

- $x_{2}(u)=\frac{1}{\omega}\left[x_{2}\left(\varphi_{0}\right)-2 k \cos (\phi) \mathrm{cn}(u)+(u-2 E(u)) \sin (\phi)\right]$.

- $x_{3}(u)=\frac{1}{\omega^{2}}\left[x_{3}\left(\varphi_{0}\right)-\sin (2 \phi)(\operatorname{sn}(u))^{2} k^{2}+k^{2} \sin (2 \phi)-1 / 4 \sin (2 \phi) u^{2}\right.$

$+\cos (2 \phi) \operatorname{cn}(u) k u-2 k x_{1}\left(\varphi_{0}\right) \cos (\phi) \operatorname{cn}(u)-2 \mathrm{E}(u) \operatorname{cn}(u) k+u \operatorname{cn}(u) k$

$-\sin (2 \phi)(\mathrm{E}(u))^{2}-2 x_{1}\left(\varphi_{0}\right) \sin (\phi) \mathrm{E}(u)+\sin (2 \phi) u \mathrm{E}(u)$

$\left.+2 \mathrm{dn}(u) k \operatorname{sn}(u)+x_{1}\left(\varphi_{0}\right) \sin (\phi) u-2 \cos (2 \phi) \operatorname{cn}(u) \mathrm{E}(u) k\right]$.

- $x_{4}(u)=\frac{1}{\omega^{3}}\left[x_{4}\left(\varphi_{0}\right)-2 \cos ^{3}(\phi) u k^{2}+2 k^{2} x_{1}\left(\varphi_{0}\right) \cos ^{2}(\phi)+4 \cos ^{3}(\phi) \mathrm{E}(u) k^{2}\right.$ $+x_{3}\left(\varphi_{0}\right) \sin (\phi) u-2 x_{3}\left(\varphi_{0}\right) \sin (\phi) \mathrm{E}(u)-\cos ^{3}(\phi) u^{2} \mathrm{E}(u)$

$+2 \cos ^{3}(\phi) u\left(\mathrm{E}(u)^{2}+\cos (\phi) u^{2} \mathrm{E}(u)-1 / 6 \cos (\phi) u^{3}-4 / 3 \cos ^{3}(\phi) \mathrm{E}(u)^{3}\right.$ 
$-1 / 2 x_{1}\left(\varphi_{0}\right) \cos ^{2}(\phi) u^{2}-2 x_{1}\left(\varphi_{0}\right) \cos ^{2}(\phi) \mathrm{E}(u)^{2}-2 \cos (\phi) u \mathrm{E}(u)^{2}$

$-2 x_{1}\left(\varphi_{0}\right) u \mathrm{E}(u)+k \sin (\phi) \operatorname{cn}(u) u^{2} \cos ^{2}(\phi)+2 \cos ^{3}(\phi) u k^{2} \operatorname{sn}(u)^{2}$

$-2 / 3 \cos (\phi) \mathrm{E}(u)+4 / 3 k^{3} \operatorname{cn}(u) \operatorname{sn}(u)^{2} \sin (\phi) \cos ^{2}(\phi)+2 k \sin (\phi) \operatorname{cn}(u)$

$+2 / 3 \cos (\phi) u-8 / 3 \cos (\phi) k^{2} \operatorname{dn}(u) \operatorname{sn}(u) \operatorname{cn}(u)-8 / 3 k^{3} \sin (\phi) \operatorname{cn}(u)$

$+4 / 3 \mathrm{E}(u) k^{2} \cos (\phi)-2 / 3 u k^{2} \cos (\phi)-4 \cos ^{3}(\phi) k^{2} \operatorname{sn}(u)^{2} \mathrm{E}(u)$

$+2 x_{1}\left(\varphi_{0}\right) \cos ^{2}(\phi) u \mathrm{E}(u)-2 x_{3}\left(\varphi_{0}\right) k \cos (\phi) \operatorname{cn}(u)+1 / 2 x_{1}\left(\varphi_{0}\right) u^{2}$

$-4 / 3 k^{3} \sin (\phi) \operatorname{cn}(u) \cos ^{2}(\phi)-2 k \sin (\phi) \operatorname{cn}(u) x_{1}\left(\varphi_{0}\right) \cos (\phi) u$

$+4 k \sin (\phi) \operatorname{cn}(u) x_{1}\left(\varphi_{0}\right) \cos (\phi) \mathrm{E}(u)+1 / 6 \cos ^{3}(\phi) u^{3}+4 / 3 \cos (\phi)\left(\mathrm{E}(u)^{3}\right.$

$-4 / 3 k^{3} \operatorname{cn}(u) \operatorname{sn}(u)^{2} \sin (\phi)-2 k^{2} x_{1}\left(\varphi_{0}\right) \cos ^{2}(\phi) \operatorname{sn}(u)^{2}+2 x_{1}\left(\varphi_{0}\right) \mathrm{E}(u)^{2}$

$\left.-4 k \sin (\phi) \operatorname{cn}(u) u \mathrm{E}(u) \cos ^{2}(\phi)+4 k \sin (\phi) \operatorname{cn}(u) \mathrm{E}(u)^{2} \cos ^{2}(\phi)\right]$.

- $x_{5}(u)=\frac{1}{\omega^{3}}\left[x_{5}\left(\varphi_{0}\right)+-4 k^{2} x_{1}\left(\varphi_{0}\right) \sin (\phi) \cos (\phi) \operatorname{sn}(u)^{2}-4 / 3 u \sin (\phi)\right.$

$+4 \cos ^{2}(\phi) \sin (\phi) u k^{2} \operatorname{sn}(u)^{2}-8 \cos ^{2}(\phi) \sin (\phi) k^{2} \operatorname{sn}(u)^{2} \mathrm{E}(u)$

$+4 x_{1}\left(\varphi_{0}\right) \cos ^{2}(\phi) u k \operatorname{cn}(u)-8 x_{1}\left(\varphi_{0}\right) \cos ^{2}(\phi) \mathrm{E}(u) k \operatorname{cn}(u)$

$+8 k \cos (\phi) \mathrm{E}(u) \operatorname{sn}(u) \operatorname{dn}(u)-4 k \cos (\phi) u \operatorname{sn}(u) \operatorname{dn}(u)$

$+8 k \cos ^{3}(\phi) \operatorname{cn}(u) u \mathrm{E}(u)+4 x_{1}\left(\varphi_{0}\right) \sin (\phi) \cos (\phi) u \mathrm{E}(u)$

$+4 k^{2} x_{1}\left(\varphi_{0}\right) \sin (\phi) \cos (\phi)+8 \cos ^{2}(\phi) \sin (\phi) \mathrm{E}(u) k^{2}-8 / 3 \sin (\phi) \mathrm{E}(u) k^{2}$

$-4 \cos ^{2}(\phi) \sin (\phi) u k^{2}-2 k \cos ^{3}(\phi) \operatorname{cn}(u) u^{2}-8 k \cos ^{3}(\phi) \operatorname{cn}(u) \mathrm{E}(u)^{2}$

$+4 \sin (\phi) \cos ^{2}(\phi) u \mathrm{E}(u)^{2}-2 \sin (\phi) \cos ^{2}(\phi) u^{2} \mathrm{E}(u)-x_{1}\left(\varphi_{0}\right) \cos (\phi) \sin (\phi) u^{2}$

$-2 x_{1}\left(\varphi_{0}\right)^{2} k \cos (\phi) \operatorname{cn}(u)-4 x_{1}\left(\varphi_{0}\right) \sin (\phi) \cos (\phi)\left(\mathrm{E}(u)^{2}+4 k \cos (\phi) \operatorname{cn}(u)\right.$

$-8 k^{3} \cos (\phi) \operatorname{cn}(u)-2 x_{1}\left(\varphi_{0}\right)^{2} \sin (\phi) \mathrm{E}(u)+1 / 3 \sin (\phi) \cos ^{2}(\phi) u^{3}$

$-8 / 3 \sin (\phi) \cos ^{2}(\phi) \mathrm{E}(u)^{3}+x_{1}\left(\varphi_{0}\right)^{2} \sin (\phi) u-8 / 3 k^{3} \cos ^{3}(\phi) \operatorname{cn}(u) \operatorname{sn}(u)^{2}$

$+4 / 3 \mathrm{E}(u) \sin (\phi)+4 / 3 \sin (\phi) u k^{2}-8 / 3 k^{2} \operatorname{dn}(u) \operatorname{sn}(u) \operatorname{cn}(u) \sin (\phi)$

$\left.+4 x_{1}\left(\varphi_{0}\right) k \operatorname{sn}(u) \operatorname{dn}(u)+8 / 3 k^{3} \cos ^{3}(\phi) \operatorname{cn}(u)\right]$.

\section{Rotating case}

We can perform the same computations as in the oscillating case.

Abnormal case. According to [7], we consider the minimal time problem for the single-input affine system

$$
\dot{x}(t)=\hat{F}_{1}(x(t))+u(t) \hat{F}_{2}(x(t))
$$


where $u$ is a scalar control.

Denoting $x($.$) a reference minimum time trajectory, it follows from the Pontryagin$ maximum principle that along the extremal lift of $x($.$) , there must hold H_{2}(x(),. p())=$. 0 and derivating with respect to $t,\left\{H_{1}, H_{2}\right\}(x(),. p())=$.0 must hold too. Thanks to a further derivation, the extremals associated with the controls

$$
u_{a}(x, p)=\frac{\left\{H_{1},\left\{H_{2}, H_{1}\right\}\right\}(x, p)}{\left\{H_{2},\left\{H_{1}, H_{2}\right\}\right\}(x, p)}=\frac{2 p_{5}}{p_{4}}
$$

satisfy the constraints $H_{2}=\left\{H_{1}, H_{2}\right\}=0$ along $(x(),. p()$.$) and are solutions of$

$$
\dot{x}=\frac{\partial H_{a}}{\partial p}, \quad \dot{p}=-\frac{\partial H_{a}}{\partial x}
$$

where $H_{a}$ is the true Hamiltonian

$$
H_{a}(x, p)=H_{1}(x, p)+u_{a} H_{2}(x, p)=p_{1}+2 \frac{p_{5}\left(p_{2}+p_{3} x_{1}+p_{4} x_{3}+p_{5} x_{1}^{2}\right)}{p_{4}} .
$$

We consider the abnormal extremals that is the constraint $H_{1}(x(),. p())=$.0 must hold. The extremal system subject to the constraints $H_{1}=H_{2}=\left\{H_{1}, H_{2}\right\}=0$ is integrable and solutions can be written as

$$
\begin{aligned}
& x_{1}(t)=t+x_{1}(0), \quad x_{2}(t)=2 \frac{p_{5}(0) t}{p_{4}(0)}+x_{2}(0), \\
& x_{3}(t)=\frac{p_{5}(0) t^{2}}{p_{4}(0)}+2 \frac{p_{5}(0) x_{1}(0) t}{p_{4}(0)}+x_{3}(0), \\
& x_{4}(t)=2 / 3 \frac{p_{5}(0)^{2} t^{3}}{p_{4}(0)^{2}}-2 \frac{p_{5}(0)\left(p_{5}(0) x_{1}(0)^{2}+p_{3}(0) x_{1}(0)+p_{2}(0)\right) t}{p_{4}(0)^{2}} \\
&-\frac{p_{5}(0) p_{3}(0) t^{2}}{p_{4}(0)^{2}}+x_{4}(0), \quad p_{4}(0) \\
& x_{5}(t)=2 / 3 \frac{p_{5}(0) t^{3}}{p_{4}(0)}+\frac{\left(4 p_{5}(0) x_{1}(0)+p_{3}(0)\right) t^{2}}{p_{4}(0)} \\
&\left.+2 \frac{\left(2 p_{5}(0) x_{1}(0)^{2}+p_{3}(0) x_{1}(0)+x_{3}(0) p_{4}(0)+p_{2}(0)\right) t}{p_{4}(0)}\right) t+p_{1}(0), \\
& p_{1}(t)=\left(-2 \frac{p_{5}(0) p_{3}(0)}{p_{4}(0)}-4 \frac{p_{5}(0)^{2} x_{1}(0)}{p_{3}(t)=-2 p_{5}(0) t+p_{3}(0), \quad p_{4}(t)=p_{4}(0),} \quad p_{5}(t)=p_{5}(0)\right. \\
& p_{2}(t)=p_{2}(0), \quad p_{1}(0)=0, p_{2}(0)=p_{5}(0) x_{1}(0)^{2}-p_{3}(0), p_{3}(0)=-2 p_{5}(0) x_{1}(0) . \\
& \text { with }\left(x_{1}(0), x_{2}(0), x_{3}(0), x_{4}(0), x_{5}(0), p_{1}(0), p_{2}(0), p_{3}(0), p_{4}(0), p_{5}(0)\right) \text { are constants } \\
& \text { satisfying }
\end{aligned}
$$




\section{Numerical results}

This section presents the numerical simulations performed on the Purcell swimmer problem. Simulations are performed using both direct and indirect methods, using the solvers BOCOP and НАMPATH. We use the multipliers from the solutions of the direct method to initialize the costate variables in the indirect approach. We show the optimal trajectories obtained for the nilpotent approximation and the true mechanical system.

Bocop. Bocop (www . bocop.org, [4]) implements a so-called direct transcription method. Namely, a time discretization is used to rewrite the optimal control problem as a finite dimensional optimization problem (i.e nonlinear programming), solved by an interior point method (IPOPT). We recall below the optimal control problem, formulated with the state $q=\left(\alpha_{1}, \alpha_{2}, x, y, \theta\right)$ and control $u=\left(\dot{\alpha}_{1}, \dot{\alpha}_{2}\right)$

$$
\left\{\begin{array}{l}
\operatorname{Min} \int_{0}^{T} E(u(t)) d t \\
\dot{q}(t)=F_{1}(q(t)) u_{1}(t)+F_{2}(q(t)) u_{2}(t) \\
\alpha_{1 \mid 2}(t) \in[-a, a] \\
x(0)=y(0)=0, x(T)=x_{f} \\
y(T)=y_{f}, \theta(T)=\theta(0), \alpha_{1 \mid 2}(T)=\alpha_{1 \mid 2}(0)
\end{array}\right.
$$

НамРАтн. The HamPATH software (http://cots . perso.enseeiht.fr/hampath/, [9]) is based upon indirect methods to solve optimal control problems using simple shooting methods and testing the local optimality of the solutions.

More precisely two purposes are achieved with НАMРАTH:

- Shooting equations: to compute periodic trajectories of the Purcell swimmer, we consider the true Hamiltonian $H$ given by the Pontryagin maximum principle and the transversality conditions associated with. The normal and regular minimizing curves are the projection of extremals solutions of the boundary two values problem

$$
\left\{\begin{array}{l}
\dot{q}=\frac{\partial H}{\partial p}, \quad \dot{p}=-\frac{\partial H}{\partial q}, \\
x(0)=x_{0}, \quad x(T)=x_{f}, \quad y(0)=0, \quad y(T)=y_{f} \\
\alpha_{1 \mid 2}(T)=\alpha_{1 \mid 2}(0), \quad \theta(T)=\theta(0), \\
p_{\alpha_{1 \mid 2}}(T)=p_{\alpha_{1 \mid 2}}(0), \quad p_{\theta}(T)=p_{\theta}(0) .
\end{array}\right.
$$

where $q=\left(x, y, \alpha_{1}, \alpha_{2}, \theta\right), p=\left(p_{x}, p_{y}, p_{\alpha_{1}}, p_{\alpha_{2}}, p_{\theta}\right)$ and $T>0$ is fixed.

Due to the sensitivity of the initialization of the shooting algorithm, the latter is initialized with direct methods namely the BOCOP toolbox.

- Local optimality: to show that the normal stroke is optimal we perform a rank test on the subspaces spanned by solutions of the variational equation with suitable initial conditions [7]. 


\subsection{Nilpotent approximation}

Notations: $\quad$ state $x=\left(x_{1}, x_{2}, x_{3}, x_{4}, x_{5}\right)$, costate $p=\left(p_{1}, p_{2}, p_{3}, p_{4}, p_{5}\right), \hat{F}_{1}, \hat{F}_{2}$ the normal form given by (4.1), and $H_{1}, H_{2}$ are the respective Hamiltonian lifts.

Normal case. In the normal case, we consider the extremal system given by the true Hamiltonian given by (4.2). We compute the optimal trajectories with HAMPATH, and show the state and adjoint variables as functions of time on Fig.2. We also illustrate the conjugate points computed according to the algorithm in [6], as well as the smallest singular value for the rank test.
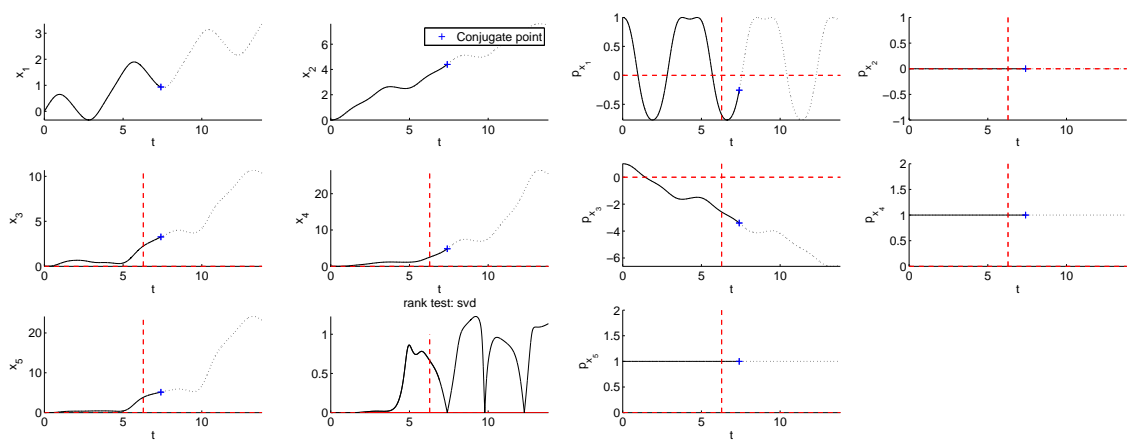

Figure 2: Nilpotent approximation (normal case): state, adjoint variables and first conjugate point (blue cross), with the smallest singular value of the rank test.

Property on the first conjugate point. Let us consider the fixed energy level $H_{1}(t=$ $0)^{2}+H_{2}(t=0)^{2}=1$ along the extremals and the initial state $x(0)=0$. We take a large number of random initial adjoint vectors $p(0)$ and numerically integrate the extremal system. For each normal extremal, we compute the first conjugate time $t_{1 c}$, the pulsation $\omega=\left(p_{4}(0)^{2}+4 p_{5}(0)^{2}\right)^{1 / 4}$, and the complete elliptic integral $K(k)$, where $k$ is the amplitude

$$
k=\frac{1}{2} \sqrt{\frac{2 \sqrt{p_{4}(0)^{2}+4 p_{5}(0)^{2}}+p_{3}(0)^{2}-2 p_{1}(0) p_{4}(0)-4 p_{5}(0) p_{2}(0)-4 p_{5}(0) p_{4}(0) x_{3}(0)}{\sqrt{p_{4}(0)^{2}+4 p_{5}(0)^{2}}}} .
$$

Let $\gamma($.$) be a normal extremal starting at t=0$ from the origin and defined on $[0,+\infty[$. As illustrated on Fig.3, there exist a first conjugate point along $\gamma$ corresponding to a conjugate time $t_{1 c}$ satisfying the inequality:

$$
0.3 \omega t_{1 c}-0.4<K(k)<0.5 \omega t_{1 c}-0.8 \text {. }
$$

Remark 5.1. In section $4.2 u=\omega t+\varphi_{0}$ is the normalised parametrization of the solutions. 


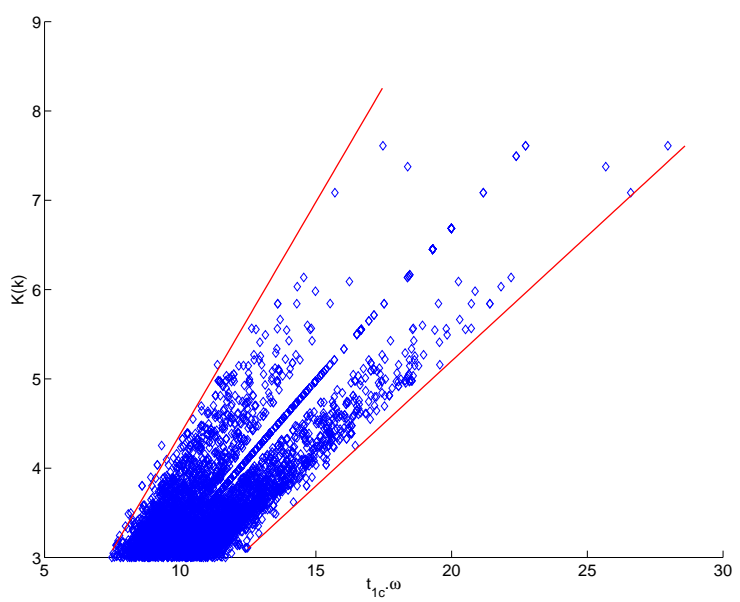

Figure 3: Normal extremals with constant energy $H_{1}^{2}+H_{2}^{2}=1$. The first conjugate point on the elliptic integral $K\left(k, \omega t_{c}\right)$ satisfies $0.3 \omega t_{1 c}-0.4<K(k)<0.5 \omega t_{1 c}-0.8$. Illustration for random initial costate $p(0)$.

Abnormal case. Fig.4 illustrates the time evolution of the state variables. We check the second order optimality conditions thanks to the algorithm given in [7]. The determinant test and smallest singular value for the rank condition both indicate that there is no conjugate time for abnormal extremals (Fig.5).
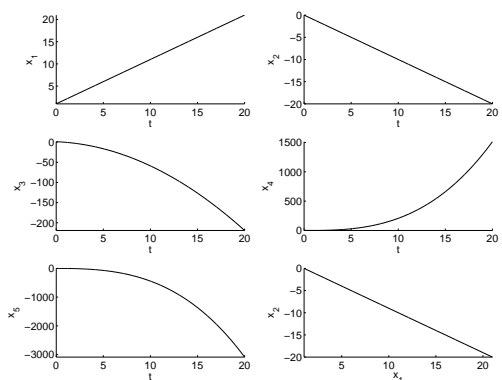

Figure 4: Abnormal case: state variables for $x(0)=(1,0,1,0,0), p(0)=(0,0,-2,1,1)$.
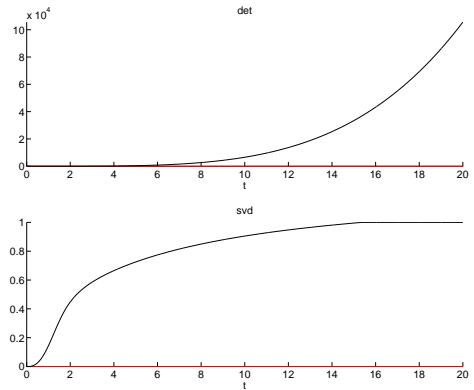

Figure 5: Abnormal case: the second order sufficient condition indicates there is no conjugate point.

\subsection{True mechanical system}

We now consider the optimal control problem (5.1) consisting in minimizing either the mechanical energy (3.1) or the criterion $|u|^{2}$.

Direct method. In the first set of simulations performed by Bocop, we set $T=$ $10, x_{f}=0.5$, and the bounds $a=3$ large enough so that the solution is actually 
unconstrained. The state and control variables for the optimal trajectory are shown on Fig.6, 7 and 8, and we observe that the trajectory is actually a sequence of identical strokes. Fig.9 shows the phase portrait for the shape angles $\alpha_{1}, \alpha_{2}$, which is an ellipse. The constant energy level satisfied by the optimal trajectory means the phase portrait of the controls is a circle for the $|u|^{2}$ criterion, but not for the energy criterion. The adjoint variables (or more accurately in this case, the multipliers associated to the discretized dynamics) are shown on Fig.10-11.
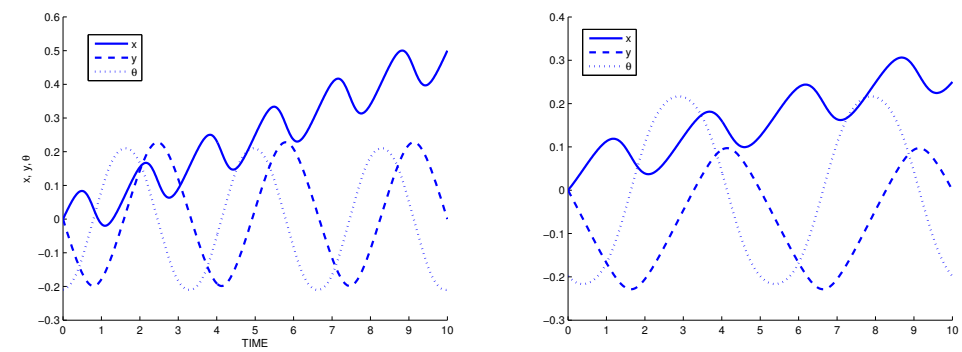

Figure 6: Optimal trajectory for $|u|^{2}$ and energy criterion - state variables $x, y, \theta$
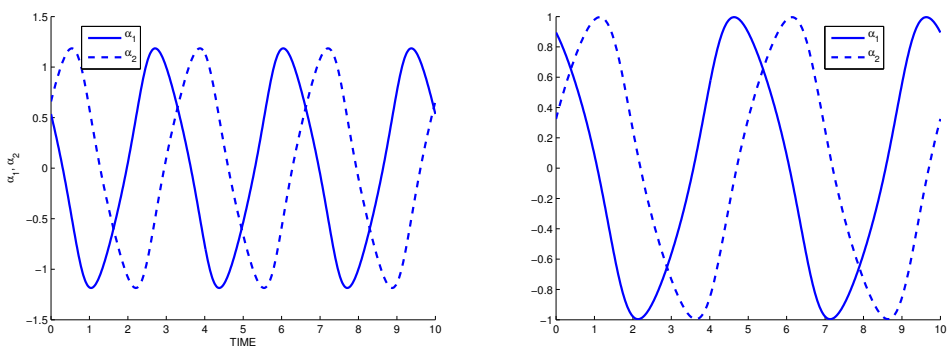

Figure 7: Optimal trajectory for $|u|^{2}$ and energy criterion - state variables $\alpha_{1}, \alpha_{2}$
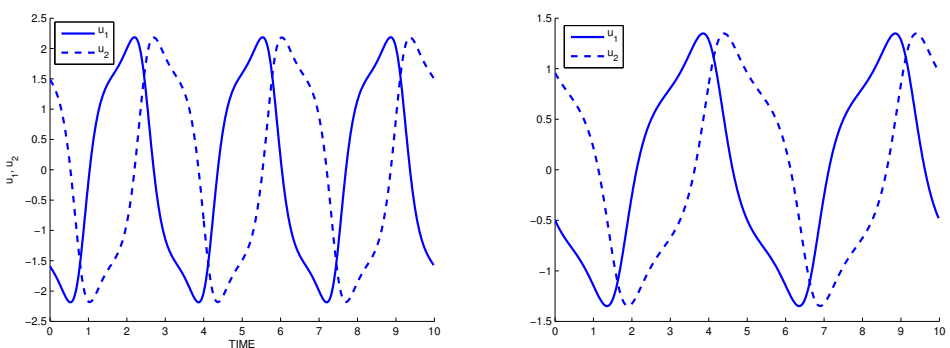

Figure 8: Optimal trajectory for $|u|^{2}$ and energy criterion - control variables

Indirect method. Now we use the multipliers from the BOCOP solutions to initialize the shooting algorithm of HAMPATH. Fig.12-13 and Fig.14-15 represent respectively an non intersecting curve and an eight shape curve with the same boundary values. 

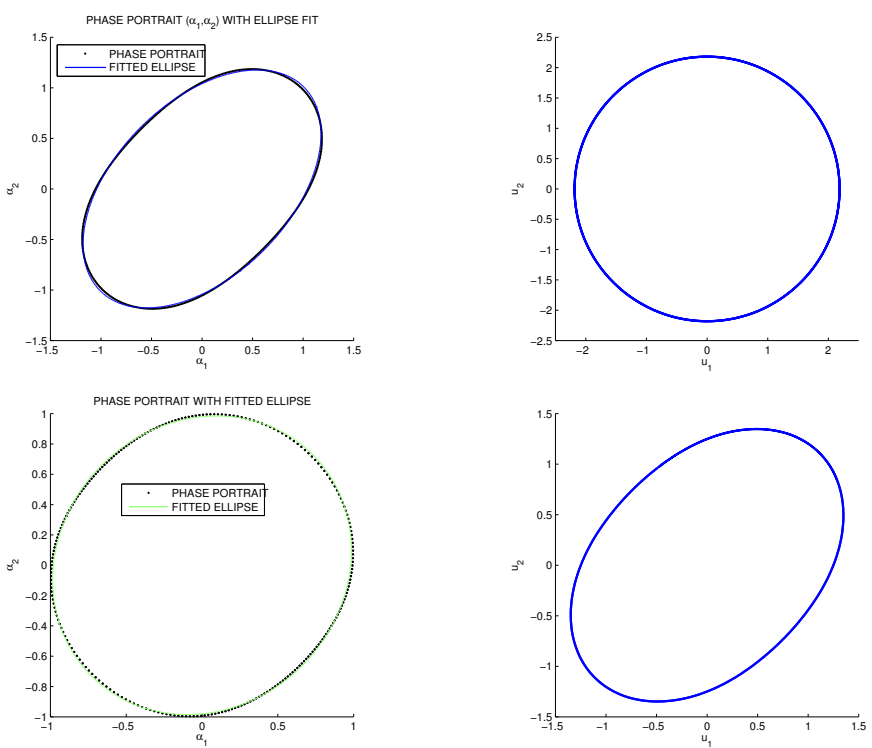

Figure 9: Optimal trajectory for $|u|^{2}$ and energy criterion - Phase portrait (ellipse) and controls
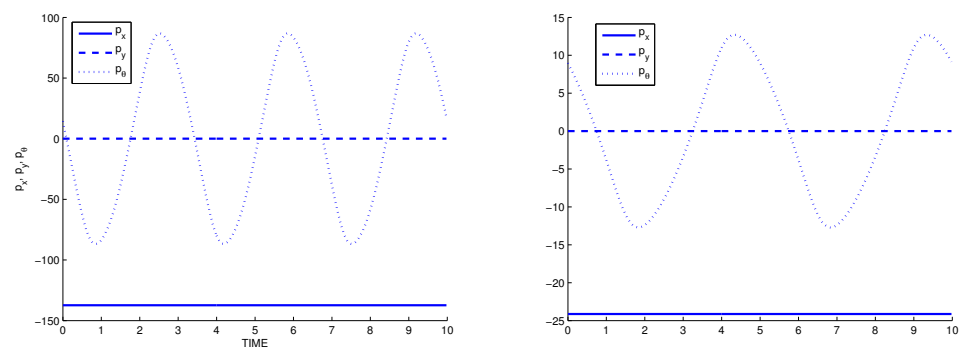

Figure 10: Optimal trajectory for $|u|^{2}$ and energy criterion - adjoint variables
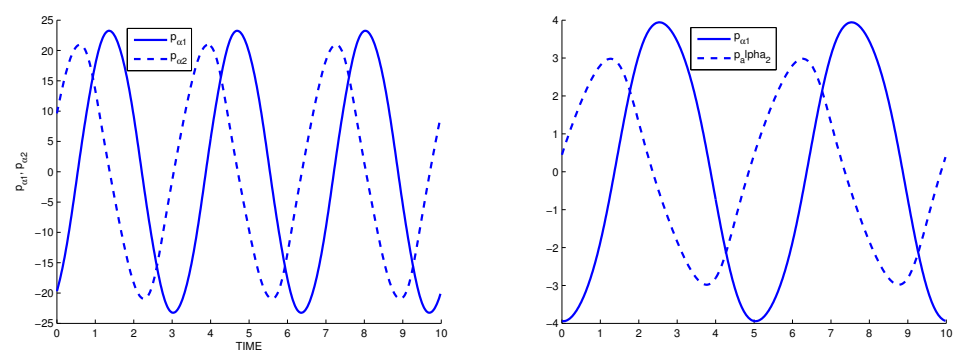

Figure 11: Optimal trajectoryfor $|u|^{2}$ and energy criterion - adjoint variables $p_{\alpha_{1}}, p_{\alpha_{2}}$ 
Fig.16-17 shows another eight shape curve obtained for different boundary values. In this three cases, we check the second order optimality conditions according to [6] and observe that there is no conjugate point on $[0, T]$ where $T=2 \pi$.

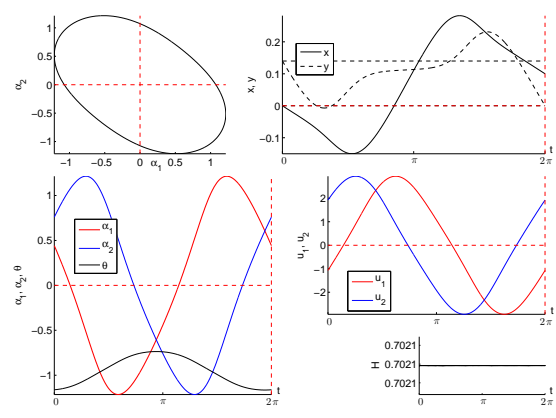

Figure 12: Non self-intersecting solution for the $|u|^{2}$ criterion $\left(x_{0}=0, y_{0}=0.14, x_{f}=0.1, y_{f}=0\right)$.

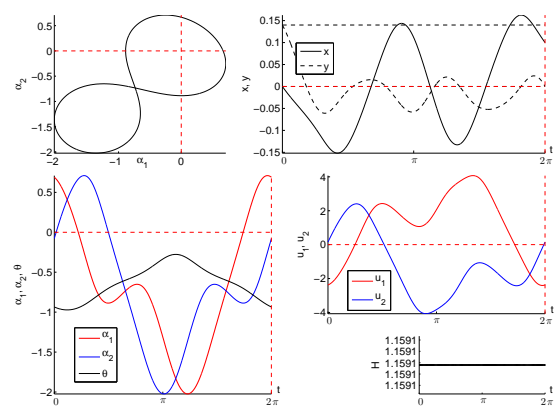

Figure 14: Solution 8-SONE for the $|u|^{2}$ criterion $\left(x_{0}=0, y_{0}=0.14, x_{f}=0.1, y_{f}=0\right)$.
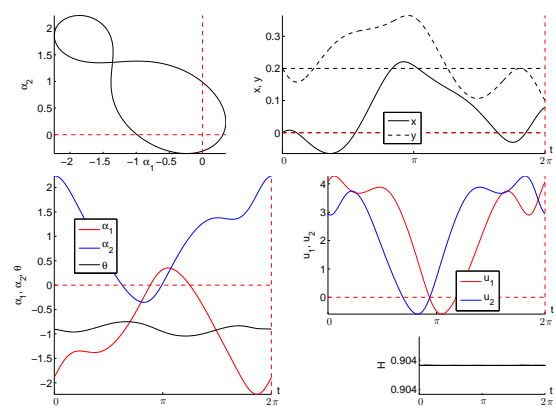

Figure 16: Solution 8-NOSE for the $|u|^{2}$ criterion $\left(x_{0}=0, y_{0}=0.2, x_{f}=0.08, y_{f}=0.1\right)$.
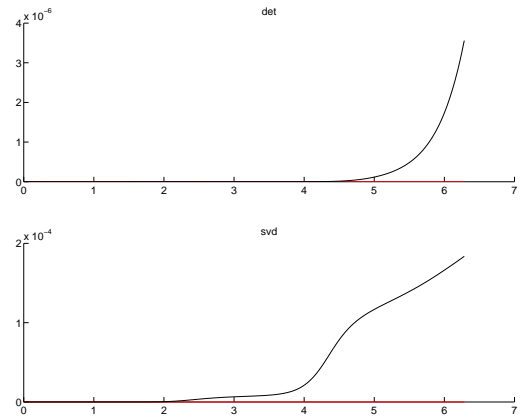

Figure 13: Second order conditions: no conjugate time $t_{1 c} \in[0,2 \pi]$.
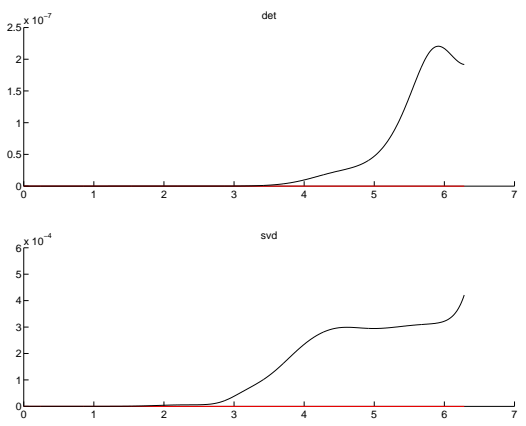

Figure 15: Second order conditions: no conjugate time $t_{1 c} \in[0,2 \pi]$.
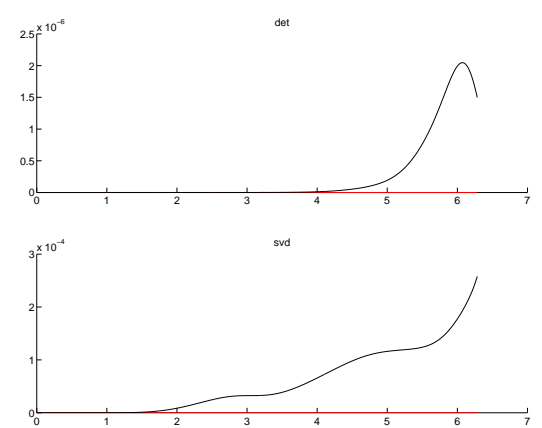

Figure 17: Second order conditions: no conjugate time $t_{1 c} \in[0,2 \pi]$. 


\subsection{The Purcell swimmer in a round swimming pool}

Clearly, due to the symmetry with respect to the initial orientation of the body, we have the following result.

Lemma 5.2. If $\alpha(t), \theta(t), \bar{x}(t), \bar{y}(t)$ is an extremal solution associated to $u($.$) with$ $\theta(0)=0$, then

$$
\begin{aligned}
& x(t)=\cos \left(\theta_{0}\right) \bar{x}(t)-\sin \left(\theta_{0}\right) \bar{y}(t), \\
& y(t)=\sin \left(\theta_{0}\right) \bar{x}(t)+\cos \left(\theta_{0}\right) \bar{y}(t)
\end{aligned}
$$

is the solution associated to $u($.$) with \theta(0)=\theta_{0},(x(0), y(0))=\left(x_{0}, y_{0}\right)$ and with the same cost $\left(|u|^{2}\right.$ criterion or energy case).

Remark 5.3. This leads to define a one parameter family of isocost extremals starting from any point. Practically this justify the following numerical computation.

Minimizers having the circle as a right end-point constraint.

We present now simulations of the following boundary value problem (BVP)

$$
\left\{\begin{array}{l}
\dot{q}=\frac{\partial H}{\partial p}, \quad \dot{p}=-\frac{\partial H}{\partial q} \\
x(0)=0, \quad y(0)=0, \quad x(T)^{2}+y(T)^{2}-R^{2}=0 \\
\alpha_{1 \mid 2}(T)=\alpha_{1 \mid 2}(0), \quad \theta(T)=\theta(0) \\
p_{\alpha_{1 \mid 2}}(T)=p_{\alpha_{1 \mid 2}}(0), \quad p_{\theta}(T)=p_{\theta}(0) \\
p_{x}(T) y(T)-p_{y}(T) x(T)=0
\end{array}\right.
$$

where $H(q, p)$ is the true Hamiltonian for the $|u|^{2}$ criterion, $q=\left(x, y, \alpha_{1}, \alpha_{2}, \theta\right)$, $p=\left(p_{x}, p_{y}, p_{\alpha_{1}}, p_{\alpha_{2}}, p_{\theta}\right)$ and $T>0$ is fixed.

For numerical simulations we set $T=2 \pi$ and $R=0.1$. Fig.18-19 show an optimal trajectory, with the test rank for the second order optimality conditions indicating that there is no conjugate time. Fig.20 represents the projection in the plane $(x, y)$ of two trajectories for different initial conditions, with the end-point circle constraint drawn in black line.

It turns out that this problem has a particular symmetry, which, taking the initial position angle $\theta_{0}$ as a parameter, allows to embed minimizers in a (one-parameter) family of minimizers. As a particular consequence we obtain the non-uniqueness of minimizers. 


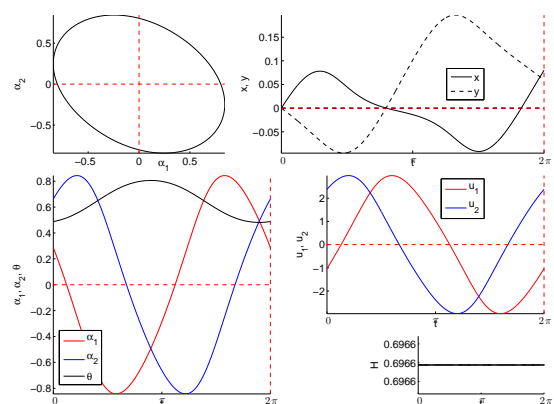

Figure 18: Circle end-point constraint: Optimal trajectory - state and control variables
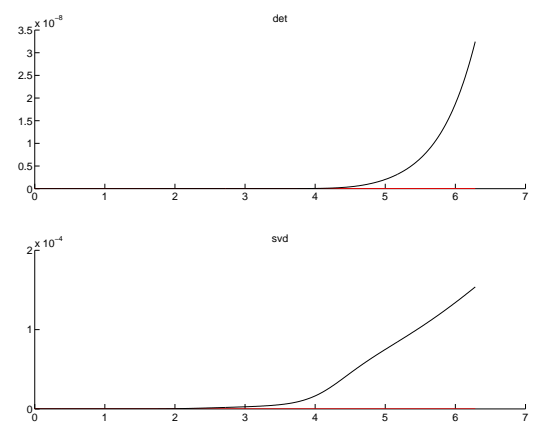

Figure 19: Determinant and smallest singular value problem of the rank condition.

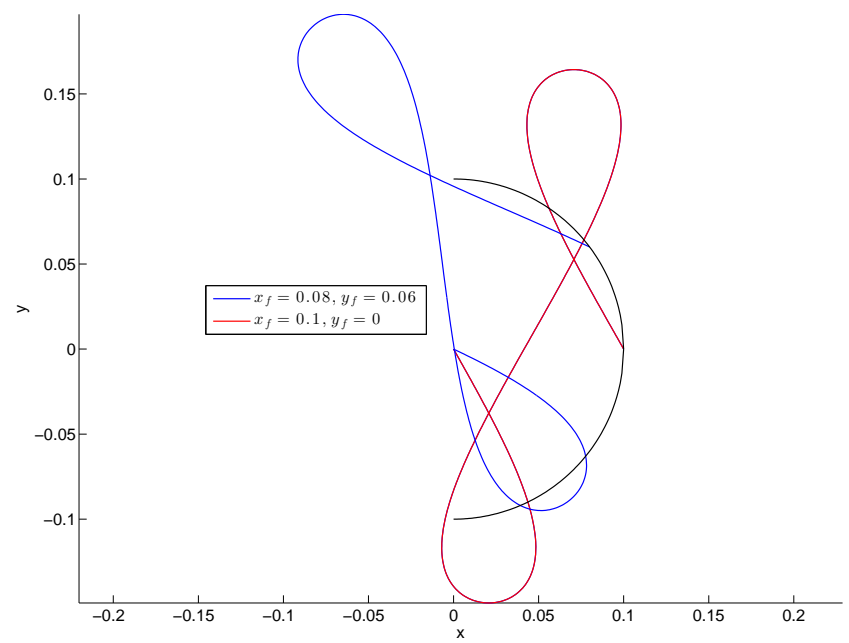

Figure 20: Circle end-point constraint: projections of two trajectories in the plane $(x, y)$.

\section{Conclusions and future work}

In the present paper we focus on some aspects related to first and second order optimality conditions applied to a mathematical model of the Purcell Three-link swimmer. Combining numerical methods with a geometrical approach we investigate crucial features of this model, as its nilpotent approximation, the integrability of extremals, the periodicity of minimizers, providing and estimate of conjugate points for both normal and abnormal extremals. This model exhibits particular properties (such as symmetries) which make it a very good case study to investigate further (non-trivial) features concerning second order optimality conditions, when non-unique minimizers occur. 


\section{Bibliography}

[1] F. Alouges, A. DeSimone and A. Lefebvre, Optimal strokes for low Reynolds number swimmers: an example, J. Nonlinear Sci. 18 (2008), pp. 277-302.

[2] F. Alouges, A. DeSimone, L. Giraldi and M. Zoppello, Self-propulsion of slender microswimmers by curvature control: N-link swimmers, Int. J. Nonlinear Mech. 56 (2013), pp. 132-141.

[3] A. Bellaïche, The tangent space in sub-Riemannian geometry, J. Math. Sci. 35 (1997), pp. 461-476.

[4] F. Bonnans, D .Giorgi, S. Maindrault, P. Martinon and V. Grélard, Bocop - A collection of examples, Inria Research Report, Project-Team Commands, 8053 (2014).

[5] B. Bonnard and M. Chyba, Singular trajectories and their role in control theory, Mathématiques \& Applications, Springer-Verlag, Berlin, 40 (2003).

[6] B. Bonnard, L. Faubourg and E. Trélat, Mécanique céleste et contrôle des véhicules spatiaux, Mathématiques \& Applications, Springer-Verlag, Berlin, 51 (2006).

[7] B. Bonnard, J.-B. Caillau and E. Trélat, Second order optimality conditions in the smooth case and applications in optimal control, ESAIM Control Optim. Calc. Var. 13 (2007), pp. 207-236.

[8] E. Cartan, Les systèmes de Pfaff a cinq variables et les équations aux derivées partielles du second ordre, Ann. Sci. École Normale 27 (1910), pp. 109-192.

[9] O. Cots. Contrôle optimal géométrique : méthodes homotopiques et applications, Phd thesis, Institut Mathématiques de Bourgogne, Dijon, France (2012).

[10] C. Gavriel and R.B. Vinter, Second order sufficient conditions for optimal control problems with non-unique minimizers: an abstract framework, Appl. Math. Optim. 70 (2014), pp. 411-442.

[11] L. Giraldi, P. Martinon and M. Zoppello, Controllability and Optimal Strokes for N-link Micro-swimmer Proc. 52th Conf. on Dec. and Contr. (Florence, Italy), (2013).

[12] L. Giraldi, P. Martinon and M. Zoppello, Optimal design of Purcell's three-link swimmer Physical Review, 91 (2015).

[13] J. Gray and G. J. Hancock, The propulsion of sea-urchin spermatozoa, Journal of Experimental Biology, 32 (1955), pp. 802-814.

[14] F. Grognard, A.R. Akhmetzhanov and O. Bernard, Periodic optimal control for biomass productivity maximization in a photobioreactor using natural light, Inria Research Report, Project-Teams Biocore, 7929 (2012).

[15] E. Kanso and P. K. Newton, Locomotory advantages to flapping out of phase, J. Exp. Mechanics, 50 (2009).

[16] D.F. Lawden, Elliptic functions and applications, Applied Mathematical Sciences, Springer-Verlag, New York, 80 (1989).

[17] A. Najafi and R. Golestanian, Simple swimmer at low Reynolds number: Three linked spheres Physical Review, 69 (2004). 
[18] H. Poincaré, CEuvres. Tome VII, Éditions Jacques Gabay, Sceaux, (1996).

[19] L. S. Pontryagin, V. G. Boltyanskii, R.V. Gamkrelidze and E. F.Mishchenko, The mathematical theory of optimal processes, Interscience Publishers John Wiley \& Sons, Inc. New York-London (1962).

[20] E. M. Purcell, Life at low Reynolds number, Am. J. Phys. (1977), pp. 3-11.

[21] Y.L. Sachkov, Symmetries of flat rank two distributions and sub-Riemannian structures, Trans. Amer. Math. Soc. 356 (2004), pp. 457-494.

[22] J.L. Speyer and R.T. Evans, A second variational theory for optimal periodic processes, IEEE Trans. Automat. Control 29 (1984), pp. 138-148.

[23] G.I. Taylor. Analysis of the swimming of microscopic organisms. Proc. Roy. Soc. London. Ser. A. 209 (1951), pp. 447-461.

[24] Q. Wang and J.L. Speyer, Necessary and sufficient conditions for local optimality of a periodic process, SIAM J. Control Optim. 28 (1990), pp. 482-497.

\section{Author information}

Piernicola Bettiol, Laboratoire de Mathématiques Unité CNRS UMR 6205,

Université de Bretagne Occidentale,

6, Avenue Victor Le Gorgeu,

29200 Brest, France.

E-mail: piernicola.bettiol@univ-brest.fr

Bernard Bonnard, Inria Sophia Antipolis et Institut de Mathématiques de Bourgogne,

9 avenue Savary,

21078 Dijon, France.

E-mail: bernard.bonnard@u-bourgogne.fr

Laetitia Giraldi, ENSTA, Paristech

828, Boulevard des Maréchaux,

91762 Palaiseau, France.

E-mail: laetitia.giraldi@ensta-paristech.fr

Pierre Martinon, Inria Saclay et CMAP Ecole Polytechnique,

Route de Saclay,

91128 Palaiseau, France.

E-mail: pierre.martinon@inria.fr

Jérémy Rouot, Inria Sophia Antipolis,

2004 route des lucioles,

F-06902 Sophia Antipolis, France.

E-mail: jeremy.rouot@inria.fr 